Revue d'histoire de l'Amérique française

DEVUE D.HISTOIRE DE L'AMÉRIQUE FRANÇAISE

\title{
Un territoire de cent pas de côté : récits de la création d'une réserve indienne en territoire innu au milieu $\mathrm{du} \mathrm{XX}^{\mathrm{e}}$ siècle
}

\author{
Aude Maltais-Landry
}

Volume 69, numéro 1-2, été-automne 2015

URI : https://id.erudit.org/iderudit/1034588ar

DOI : https://doi.org/10.7202/1034588ar

Aller au sommaire du numéro

\section{Éditeur(s)}

Institut d'histoire de l'Amérique française

\section{ISSN}

0035-2357 (imprimé)

1492-1383 (numérique)

Découvrir la revue

\section{Citer cet article}

Maltais-Landry, A. (2015). Un territoire de cent pas de côté : récits de la création d'une réserve indienne en territoire innu au milieu du $\mathrm{XX}^{\mathrm{e}}$ siècle. Revue d'histoire de l'Amérique française, 69(1-2), 19-50.

https://doi.org/10.7202/1034588ar
Résumé de l'article

Cet article se base sur des récits recueillis dans le cadre d'un projet de recherche en histoire orale mené avec des membres de la communauté innue de Nutashkuan, sur la Côte-Nord, et qui portait sur la création de leur réserve dans les années 1950. Il se veut un exemple d'une utilisation possible de l'histoire orale où les récits, placés au coeur de la trame narrative, permettent d'écrire une histoire des Autochtones qui soit portée par leur propre compréhension des événements. Au-delà de leur ancrage dans des lieux très précis, les récits présentés ici ouvrent la porte à une histoire plus vaste de l'imposition de la Loi sur les Indiens aux communautés autochtones du Québec. 


\section{Un territoire de cent pas de côté : récits de la création d'une réserve indienne en territoire innu au milieu du XXe siècle}

Aude Maltais-Landry

Centre d'histoire orale et de récits numérisés

Université Concordia

RÉSUMÉ - Cet article se base sur des récits recueillis dans le cadre d'un projet de recherche en histoire orale mené avec des membres de la communauté innue de Nutashkuan, sur la Côte-Nord, et qui portait sur la création de leur réserve dans les années 1950. II se veut un exemple d'une utilisation possible de l'histoire orale où les récits, placés au cœur de la trame narrative, permettent d'écrire une histoire des Autochtones qui soit portée par leur propre compréhension des événements. Au-delà de leur ancrage dans des lieux très précis, les récits présentés ici ouvrent la porte à une histoire plus vaste de l'imposition de la Loi sur les Indiens aux communautés autochtones du Québec.

ABSTRACT - This article is based on stories collected during an oral history project conducted with members of the Innu community of Nutashkuan (North Shore region, Quebec), which explored the creation of their reserve in the 1950s. It argues that oral history, by putting the stories at the heart of the narrative, can allow us to write a history of Aboriginal peoples that is propelled by their own comprehension of historical events. Although locally grounded, the stories presented here shed light on the broader implications of the imposition of the Indian Act on Aboriginal communities in Quebec.

vant d'entrer dans le vif du sujet de cet article, quelques considéra-
tions disciplinaires s'imposent. D’emblée, je dirai que je préfère au

1. Je tiens à remercier l'ensemble de la communauté de Nutashkuan pour son accueil, ainsi que tous les participants au projet, que leurs noms apparaissent ou non dans ces pages. 
terme de "sources orales» celui d' «histoire orale», selon une définition largement développée dans le milieu anglo-saxon qui entrevoit l'histoire orale comme une discipline et une méthodologie à part entière ${ }^{2}$. Bien que les sources orales puissent effectivement pallier un manque de sources écrites, surtout dans le cas de groupes marginalisés et généralement exclus de l'histoire officielle ${ }^{3}$, il serait trompeur de les voir comme un simple "support» à un ensemble d'écrits ou de statistiques qui s'avérerait incomplet ou insatisfaisant. L'histoire orale nécessite de repenser le rapport aux sources, puisque celles-ci sont créées par l'historien et le narrateur, au cours d'une rencontre qui permet l'éclosion d'un récit et l'organisation de souvenirs personnels en une trame narrative cohérente ${ }^{4}$. Historien et narrateur deviennent tous deux "propriétaires " de l'histoire, dans un rapport de partage de l'autorité 5 . Ainsi, l'histoire orale diffère non seulement d'une vision de la discipline historique où les «sources orales" sont simplement d'autres "sources", mais également de la démarche de l'entrevue qualitative en sciences sociales où le récit reste le plus souvent anonyme.

Un deuxième postulat s'impose quant au sens qu'on choisit de donner à l'histoire du Québec et à l'inclusion ou non de l'histoire des Autochtones dans cette histoire. Force est de constater que le récit historique national dominant laisse encore peu de place aux Autochtones ${ }^{6}$, et que leur histoire est généralement faite "à part », dans une discipline distincte - l'ethnohistoire - et des revues séparées, ce qui a pour effet, selon certains, de perpétuer une autre forme de colonialisme ${ }^{7}$. Or, comme le soulignent les

2. Il existe une abondante littérature sur le sujet. Voir par exemple des classiques comme Paul Thompson, The Voice of the Past: Oral History (Oxford et New York, Oxford University Press, 1978), 257 p.; Alessandro Portelli, The Battle of Valle Giulia: oral history and the art of dialogue (Madison, The University of Wisconsin Press, 1997), 368 p.; Robert Perks et Alistair Thomson, dir., The Oral History Reader, 2nd edition (Londres, Routledge, 2006), 592 p. Sur le développement de l'histoire orale au Québec, notamment l'influence de la conception française des "sources orales" par rapport à une vision de l'histoire orale comme discipline à part entière, voir Jean-Philippe Warren et Steven High, «Memory of a Bygone Era: Oral History in Quebec, 1979-1986», The Canadian Historical Review, 95, 3 (2014): 433-456.

3. Paul Thompson énonce clairement cette intention dans The Voice of the Past..., op. cit.

4. A. Portelli, "Oral History as Genre», dans The Battle of Valle Giulia..., op. cit., 3-23.

5. Défini par Michael Frisch en 1990 dans A Shared Authority (Albany, State University of New York Press, 1990), 273 p., le concept d'autorité partagée met de l'avant le rôle central de la relation entre chercheurs et participants, qui définissent ensemble la façon dont sera racontée l'histoire. Depuis la parution du livre de Frisch, le concept d’autorité partagée a été abondamment discuté.

6. Voir par exemple Jocelyn Létourneau et Sabrina Moisan, «Mémoire et récit de l'aventure historique du Québec chez les jeunes Québécois d'héritage canadien-français : coup de sonde, amorce d'analyse et de résultats, questionnement", Canadian Historical Review, 85, 2 (2004): 325-356.

7. Pour Denys Delâge, «l'ethnohistoire [...] conduit à créer une fois de plus une "réserve pour les Autochtones" "; Gérard Bouchard a calculé que les sujets autochtones représentaient moins de $1 \%$ des articles parus dans la Revue d'histoire de l'Amérique française entre 1970 et 1999. D. Delâge, «L'histoire des 
coordonnateurs de ce numéro, l'intérêt de l'histoire orale réside notamment dans le fait que les récits personnels peuvent remettre en question ou complexifier les grands récits ou mythes nationaux. Il y a là un potentiel certain pour enrichir l'histoire «nationale» du Québec et y inclure le point de vue des Autochtones, même dans le cas d'événements historiques auxquels on ne les associe pas généralement ${ }^{8}$; c'est ici que je me positionne.

Finalement, comme le champ de la recherche autochtoniste a longtemps été occupé par les anthropologues et délaissé par les historiens ${ }^{9}$, il existe encore peu de réflexions d'historiens au Québec sur l'usage de l'histoire orale en contexte autochtone. Dans le cas des Innus, puisque c'est d'eux qu'il sera ici question, les anthropologues se sont d'abord intéressés à la riche tradition orale ${ }^{10}$ et au symbolisme de leurs contes et légendes, dans la lignée d'une vision structuraliste inspirée par Claude Lévi-Strauss ; les premiers travaux de Rémi Savard ou Sylvie Vincent, par exemple, $s^{\prime}$ inscrivant dans ce courant ${ }^{11}$. Confirmant que la ligne qui sépare les disciplines est bien mince, certains travaux d'anthropologues ont une grande valeur historique, comme le récit autobiographique du chasseur Michel Grégoire, recueilli par Richard Dominique, ceux de la mission de Musquaro recueillis par Sylvie Vincent et Joséphine Bacon, ou encore l'étude complexe de José Mailhot sur l'organisation sociale des Gens de Sheshatshit ${ }^{12}$.

Premières Nations, approches et orientations ", Revue d'histoire de l'Amérique française, 53, 4 (2000): 521527: G. Bouchard, La nation québécoise au futur et au passé (Montréal, VLB éditeur, 1999), cité dans Pierre Trudel, "Histoire, neutralité et Autochtones: une longue histoire... », Revue d'histoire de l'Amérique française, 53, 4 (2000): 528-540.

8. Je pense par exemple à la période de la Révolution tranquille, au cours de laquelle s'organisa un vaste mouvement politique autochtone qui entra en contradiction avec le mouvement souverainiste québécois. Sean Mills aborde brièvement cette question dans The Empire Within: Postcolonial Thought and Political Activism in Sixties Montreal (Montréal et Kingston, McGill-Queen's University Press, 2010), 5-6 et 58-61. Voir également Caroline Desbiens, Power from the North - Territory, Identity, and the Culture of Hydroelectricity in Quebec (Vancouver, UBC Press, 2014), 312 p.

9. D. Delâge, «L'histoire des Premières Nations... », loc. cit., 522-523.

10. La tradition orale fait référence aux histoires transmises de génération en génération dans une culture orale, par rapport aux récits de première main. Sur la différence existant entre les deux en contexte innu, voir Sylvie Vincent, «La tradition orale: une autre façon de concevoir le passé», dans Alain Beaulieu, Stéphan Gervais et Martin Papillon, dir., Les Autochtones et le Québec: Des premiers contacts au Plan Nord (Montréal, Presses de l’Université de Montréal, 2013), 75-91.

11. Voir Carole Lévesque, «La culture entre mémoire et sens: parcours de l’anthropologie amérindianiste québécoise ", dans Diane Lemieux, dir. et al., Traité de la culture (Sainte-Foy, Québec, Éditions de l'IQRC/Les Presses de l'Université Laval, 2002), 99; Paul Charest, "L'anthropologie sociale et culturelle et la Côte-Nord", Revue d'histoire de la Côte-Nord, 33 (2001): 3-11

12. Richard Dominique, Le langage de la chasse: Récit autobiographique de Michel Grégoire, Montagnais de Natashquan (Sillery, Presses de l’Université du Québec, 1989), 208 p. ; Sylvie Vincent et Joséphine Bacon, "Musquaro: Récits et souvenirs des Innus de Mingan, Natashquan, La Romaine et Pakuashipi», Centre 
Cependant, il existe encore peu d'études au Québec qui, à ma connaissance, font usage de l'histoire orale pour raconter des événements de l'histoire récente du point de vue des Autochtones eux-mêmes. Mentionnons les travaux de Laurent Jérôme sur la déportation des Innus de Pakuashipi vers Unamen Shipu dans les années 1960, un événement controversé qu'il a raconté en mettant l'accent sur la subjectivité et l'interprétation des narrateurs, jusque dans le vocabulaire même lié à l'événement ${ }^{13}$. Pensons aussi à l'utilisation de l'histoire orale par Denis Gagnon dans son étude du pèlerinage contemporain de Sainte-Anne-de-Beaupré, à sa façon de mettre en scène sa relation avec les participants et d'incorporer au récit leurs réactions face à sa présence ${ }^{14}$. Notons, encore une fois, qu'il s'agit là d'anthropologues.

Si je m'explique mal la résistance de la discipline historique à utiliser les récits, en revanche, je conçois que la pratique de l'histoire orale dans un contexte de travail autochtone pose des défis. Dans tout projet d'histoire orale, la longueur et la qualité du temps passé dans une communauté est un facteur clé de la réussite, mais cela semble particulièrement le cas en contexte autochtone, où des chercheurs qui ont abondamment fait usage de l'histoire orale ont souvent développé des relations sur des décennies ${ }^{15}$. À cela s'ajoutent d'autres facteurs qui demandent temps et négociation, car la réalité d'un contexte postcolonial impose de redéfinir les conditions de la recherche, de prioriser les demandes du milieu et de développer des méthodologies collaboratives et participatives qui tiennent compte d'un historique de recherche abusif pour les peuples autochtones ${ }^{16}$. Au final,

de recherche et d'analyse en sciences humaines (ssDcc), Recueil de textes remis à Parcs Canada (2000); José Mailhot, Au pays des Innus: les gens de Sheshatshit (Montréal, Recherches amérindiennes au Québec, 1993), 214 p.

13. Laurent Jérôme, "Note de recherche - "Ka atanakaniht": La "déportation" des Innus de Pakuashipi (Saint-Augustin)", Recherches amérindiennes au Québec, 41, 2-3 (2011): 175-184. Certains parlent de déménagement, d'autres de déplacement, d'autres enfin de déportation.

14. Denis Gagnon, Deux cents ans de pèlerinage : Les Mamit Innuat à Musquaro, Sainte-Anne-de-Beaupré et Sainte-Anne-d'Unamen-Shipu (1800-2000), thèse de doctorat (anthropologie), Université Laval, 2003.

15. En contexte autochtone, voir les travaux de Julie Cruikshank (plusieurs titres) et Keith Thor Carlson, The Power of Place, the Problem of Time: Aboriginal Identity and Historical Consciousness in the Cauldron of Colonialism (Toronto, University of Toronto Press, 2010), 375 p.; en contexte non autochtone, voir Alessandro Portelli et son travail de longue haleine avec le milieu ouvrier dans They Say in Harlan County: An Oral History (Oxford, Oxford University Press, 2010), 446 p.

16. Voir Linda Tuhiwai Smith, Decolonizing methodologies : research and indigenous peoples (Londres et New York, Zed Books, 2012), 256 p. ; Robin Jarvis Brownlie et Roewan Crowe, " "So Yo Want to Hear Our Ghetto Stories?” Oral History at Ndinawe Youth Resource Centre», dans Steven High, Edward Little et Thi Ry Duong, dir., Remembering Mass Violence: Oral History, New Media and Performance (Toronto, University of Toronto Press, 2014), 203-218; Protocole de recherche des Premières Nations du Québec et du Labrador, Assemblée des Premières Nations du Québec et du Labrador, 2005. 
une fois les récits recueillis, les chercheurs se heurtent encore à la difficulté de comprendre la structure narrative des récits autochtones ${ }^{17}$, et doivent éviter qu'ils ne deviennent de simples "compléments informatifs» à une vision occidentale de l'histoire ${ }^{18}$.

Malgré ces défis, l'histoire orale reste une occasion unique d'accéder à une compréhension des Autochtones de leur propre histoire. Pour ma part, c'est après de nombreux séjours dans des réserves autochtones du Québec que je me suis intéressée au processus de création même de ces réserves. Mon intérêt pour la perception de ces lieux par leurs habitants m'a naturellement guidée vers l'histoire orale. Je me suis concentrée sur la création de la réserve innue de Nutashkuan ${ }^{19}$, une communauté située sur la Côte-Nord, à $1300 \mathrm{~km}$ de Montréal, où j'avais travaillé à l'été 2012 et commencé à tisser des liens. La réserve de Nutashkuan a été créée dans les années 1950 sur un site fréquenté traditionnellement par les Innus à l'embouchure de la Grande rivière Natashquan. Là encore, si certains aspects de l'histoire économique, politique et culturelle des Innus qui ont mené à la création des réserves ont fait l'objet de recherches historiques ${ }^{20}$, la création de la réserve elle-même, comme celle des réserves contemporaines au Québec en général, reste en revanche un sujet peu documenté ${ }^{11}$.

17. Voir Andie Diane Palmer, Maps of Experience: The Anchoring of Land to Story in Secwepemc Discourse (Toronto, University of Toronto Press, 2005), 12; Julie Cruikshank, Life Lived Like a Story: Life Stories of Three Yukon Native Elders (Lincoln et Londres, University of Nebraska Press, 1990), 1-4.

18. Voir Carole Lévesque, «Les savoirs des Autochtones: questions, défis et enjeux», dans Jean-Pierre Baillargeon, dir., Transmission de la culture, petites sociétés, mondialisation (Sainte-Foy et Québec, Éditions de l'IQRC/Les Presses de l’Université Laval, 2002), 205-207; Toby Morantz, «Lire la tradition orale, écrire l'histoire crie", Anthropologie et Sociétés, 26, 2-3 (2002): 23-43.

19. La réserve de Nutahskuan est située à six kilomètres du village blanc de Natashquan, qui en est la version francisée. Le nom officiel de la réserve, selon les Affaires indiennes, est «Natashquan, réserve indienne», mais je lui préfère le toponyme innu de Nutashkuan.

20. Voir Paul Charest, "Les stratégies de chasse des Mamit Inuat», Anthropologie et Sociétés, 20, 3 (1996) : 107-128; Jacques Frenette, Mingan au XIXe siècle: Cycles annuels des Montagnais et politiques commerciales de la Compagnie de la Baie d'Hudson (Ottawa, Musée canadien des civilisations, Musées nationaux du Canada, 1986); José Mailhot, «La marginalisation des Montagnais », dans Pierre Frenette, dir., Histoire de la Côte-Nord (Sainte-Foy, Québec, Institut québécois de recherche sur la culture, 1996), 321-357; AnneMarie Panasuk et Jean-René Proulx, La résistance des Montagnais à l'usurpation des rivières à saumon par les euro-canadiens du XVII au XXe siècles, mémoire de maitrise (anthropologie), Université de Montréal, 1981; Rémi Savard, Le rire précolombien dans le Québec d'aujourd'hui (Montréal, Les Éditions l'Hexagone-Parti pris, 1977), $157 \mathrm{p}$.

21. Un récent article d'Alain Beaulieu s'arrête au XIX ${ }^{\mathrm{e}}$ siècle, tandis que Claude Gélinas, bien qu'il propose une étude intéressante de la création des réserves atikamekw, passe très rapidement sur le bloc «1870-1960» dans son ouvrage plus général sur les Autochtones au Québec. Alain Beaulieu, «La création des réserves indiennes au Québec», dans A. Beaulieu, S. Gervais et M. Papillon, dir., Les Autochtones et le Québec..., op. cit., 135-151; Claude Gélinas, «La création des réserves atikamekw en Haute-Mauricie (1895-1950), ou quand l'Indien était vraiment un Indien ", Recherches amérindiennes au Québec, 32, 2 (2002): 35-48, et Les Autochtones dans le Québec post-confédéral, 1867-1960 (Sillery, Septentrion, 2007), 28-40. 
Entre février et novembre 2013, j’ai effectué trois séjours à Nutashkuan et réalisé une quinzaine d'entrevues formelles ${ }^{22}$ avec des participants recrutés de bouche à oreille. Au fil des séjours, ma méthodologie s'est diversifiée: faisant usage de photographies, de récits déjà recueillis, d'archives trouvées après mes premiers séjours, j’ai aussi entamé une série de rencontres informelles ${ }^{23}$ avec d'autres membres de la communauté. Ces rencontres m'ont permis de faire ressortir des récits centraux à l'établissement de la réserve, souvent partagés par plusieurs membres de la communauté, que j'ai choisis de conserver pour construire une histoire "collective». J'ai ainsi commencé à cerner non seulement les conditions dans lesquelles s'était créée la réserve, mais aussi la façon dont les Innus se souvenaient de ces événements et les sentiments mitigés liés au lieu même de la réserve, décrite tour à tour comme un espace d'exclusion et d'appartenance ${ }^{24}$. Ces façons de raconter l'histoire sont devenues aussi importantes que les faits eux-mêmes; même dans le cas où les faits racontés dans les récits auraient été «incorrects», les récits étaient révélateurs du sens donné aux événements historiques par cette communautée ${ }^{5}$.

Si l'histoire orale est souvent décrite comme une méthodologie participative et collaborative, le processus d'écriture reste généralement l'affaire de l'auteur. À l'inverse de l'autobiographie, qui émane de la volonté de l'auteur/narrateur, l'histoire orale porte en elle une tension non résolue entre "ceux ou celles qui parlent» et "celui ou celle qui écrit», particulièrement dans le milieu universitaire, où la volonté de «donner une voix » se heurte aux exigences formelles du format académique et de ce qui est reconnu comme ayant une valeur historique. À la manière d'auteurs qui ont fait exploser ce cadre, en mettant l'accent sur les récits eux-mêmes et sur leur relation avec les narrateurs ${ }^{26}$, j'ai tenté de donner une large place aux récits et de raconter le contexte dans lequel j'avais entendu ces histoires, afin de me rapprocher du dialogue tel qu'il s'était

22. J'entends par là des entrevues planifiées à l'avance et enregistrées sur support numérique.

23. Certaines de ces rencontres étaient tout à fait imprévues, d'autres plus ou moins planifiées. Ces participants sont restés anonymes parce que je ne leur ai pas explicitement demandé s'ils souhaitaient être nommés.

24. Par exemple, l'utilisation des mots "ghetto», "zoo» ou "chez nous » pour décrire la réserve.

25. Alessandro Portelli, "The Death of Luigi Trastulli: Memory and the Event", dans The Death of Luigi Trastulli and Other Stories: Form and Meaning in Oral History (Albany, State University of New York Press, 1991), 1-26.

26. J. Cruikshank, Life Lived Like a Story..., op. cit.; A. Portelli, They Say in Harlan County..., op. cit.; A. D. Palmer, Maps of Experience..., op. cit.; Treaty 7 Elders and Tribal Council, avec Walter Hildebrant, Dorothy First Rider et Sarah Carter, The True Spirit and Original Intent of Treaty 7 (Montréal, McGillQueen's University Press, 1996), 432 p. 
produit sur le terrain; on notera d'ailleurs la présence constante de certains participants dans ces pages. Au cours d'un quatrième séjour dans la communauté, j'ai également partagé avec des participants les brouillons de mes écrits, afin de valider avec eux le choix des histoires et les liens que j'avais tissés entre elles.

Cet article se base sur des récits entendus et validés au cours de ce processus, et se décline en trois sections commençant chacune par un récit qui agit comme point de départ de l'analyse. La première section s'intéresse aux questions de mémoire et de pouvoir, à partir de récits très locaux faisant directement référence au site de la réserve et aux relations avec le village voisin de Natashquan. Dans la deuxième section, il est question d'une rencontre entre le chef et un fonctionnaire qui aurait mené au choix d'un site et de ses dimensions, un événement peu documenté qui ouvre la voie à une réflexion sur le sens de l'histoire orale en l'absence de sources écrites. Finalement, à partir du récit de la pêche illégale au saumon, la troisième section aborde la perception des Innus d'avoir été exclus non seulement de l'espace géographique qu'ils occupaient mais aussi d'un espace d'activité économique.

Parce qu'ils abordent des thèmes communs comme la rencontre, la négociation, le partage du territoire et le sentiment d'exclusion, ces récits nous amènent à penser la réserve comme un espace social et politique. Ils illustrent, comme l'écrivait la géographe Doreen Massey, que «places are best thought of as nets of social relations ${ }^{27}$ ». En mêlant méthodologie et contenu, cet article se veut une illustration d'une façon dont l'histoire orale, en mettant les récits au cœur de la trame narrative, peut contribuer à une histoire contemporaine des Autochtones au Québec qui soit portée par leur propre compréhension des événements.

\section{«C'ÉTAIT NOTRE TERRITOIRE À NOUS AUTRES »: LIEUX, MÉMOIRE ET POUVOIR}

Pis nous autres on s'est toujours installés là, pour la pêche à la... pour l'île Sainte-Hélène, pour... On s'était toujours installés là, je m'en souviens même pas quand est-ce que... Pis, c'était ça la mission, là. Notre mission à nous autres, c'était là, le prêtre arrivait là, le village était là, pis... (pause) Les canots étaient faits là, y avait comme un genre d'usine là, de canots, qu'y faisaient, là... Tout se faisait là, à la Pointe. Pis... La cueillette des fruits. (pause) Fait que... C'est ça, c'est le... (pause) C'était notre territoire à nous autres. (rire) (Adèle Bellefleur, 74 ans) 
Figure I

Femmes assises et campement à la Pointe Matshiteu, vers 1950

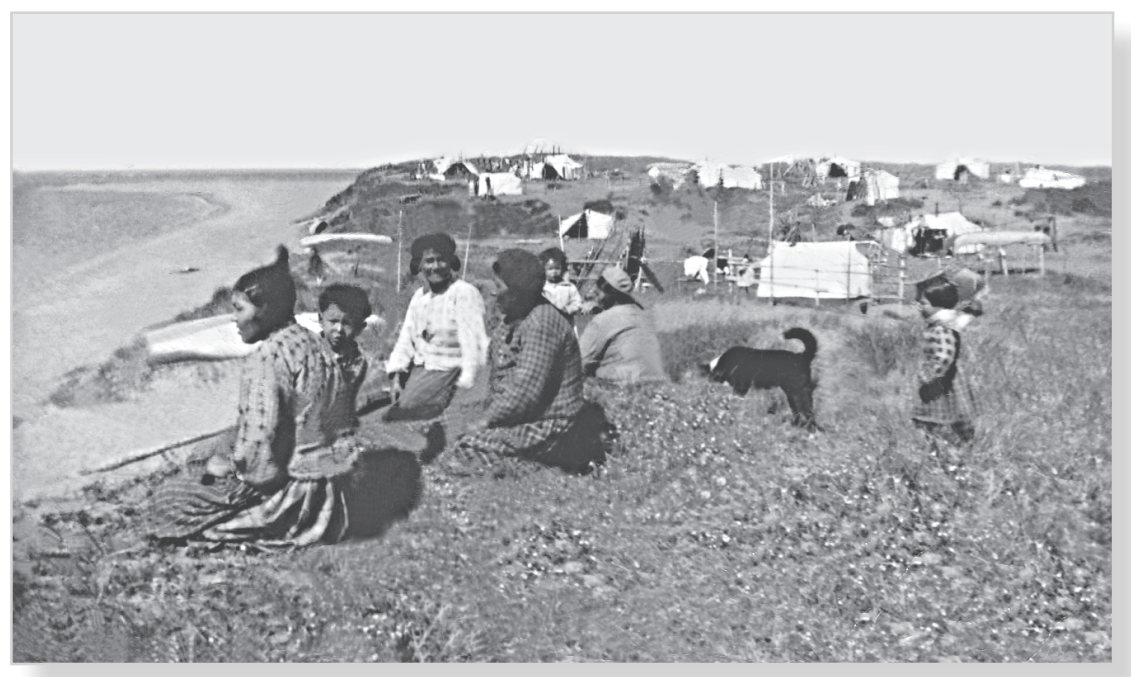

Crédit: Photo par Eleanor Leacock [?] Avec l'aimable autorisation de Pierre Wapistan.

J'ai rencontré Adèle Bellefleur à l'été 2012, alors que je travaillais à Nutashkuan avec le projet de formation audiovisuelle Wapikoni mobile. Quand j’y suis retournée en février 2013 pour présenter mon projet de recherche à la communauté, elle est l'une des premières avec qui j'ai renoué. Adèle a participé à l'élaboration des grands thèmes qui seraient abordés dans le cadre de cette recherche. Avec le temps, elle est devenue une collaboratrice essentielle, avec qui j'ai discuté à de nombreuses reprises. Cette entrevue a été enregistrée en août 2013 dans le contexte intime de son chalet, un endroit qu'elle affectionne particulièrement.

Jusque vers l'âge de 12 ans, date de son entrée au pensionnat de SeptÎles, Adèle a connu le mode de vie traditionnel des Innus, dit «nomade ${ }^{28} »$ : ils remontaient le cours des grandes rivières à saumon de la Côte-Nord à

28. L'utilisation du mot "nomade» est contestée par plusieurs chercheurs autochtones. L’idée d' "errance» associée au nomadisme, "roaming» ou "wandering" en anglais, ne correspond pas au cycle annuel très organisé des peuples autochtones dits «nomades», et a été une façon pour les Occidentaux de nier leurs droits territoriaux, notamment dans le cas Delgamuukw: voir J. Edward Chamberlin, If This Is Your Land, Where Are Your Stories? - Finding Common Ground (Manchester, Carcanet, 2006), 10. Julie Cruikshank, décrivant des déplacements cycliques très semblables à ceux des Innus, parle d'un mode de vie "flexible» (Life Lived Like A Story..., op. cit., 8-9), mais ce mot semble peu en usage en français. Faute de mieux, et parce qu'il est celui utilisé par les Innus eux-mêmes pour décrire leur mode de vie traditionnel, j'emploie à l'occasion le mot "nomade», mais pour faire référence à un mode de vie fait de déplacements cycliques structurés, nullement d’errance. 
l'automne et passaient l'hiver à l'intérieur des terres, en petits groupes familiaux. Au printemps, ils redescendaient vers la mer et se rassemblaient pour la saison estivale à l'embouchure des rivières, où ils faisaient de grandes provisions de saumon en prévision de la remontée d'automne ${ }^{29}$. À Nutashkuan, on appelle ce lieu de rassemblement Matshiteu, «la Pointe». C'est une avancée de sable à l'embouchure de Mishta Hipu, littéralement la "grande rivière » Natashquan, à l'endroit où elle se jette dans le fleuve Saint-Laurent. Matshiteu: c'est de ce lieu que parle Adèle quand elle dit: «C'était notre territoire à nous autres.»

En entrevue, Adèle a beaucoup parlé de la Pointe, et son énumération des activités qui s'y pratiquaient évoque une vie sociale complexe. Chaque été, selon son récit, les familles s'installaient sur un site près de la rivière et montaient leurs tentes au même endroit que l’année précédente ${ }^{30}$. Les retrouvailles entre les familles étaient l'occasion de célébrations diverses - makushans $s^{31}$, baptêmes, mariages ${ }^{32}$, partage des produits de la chasse, etc. La vie s'organisait autour des feux, et les femmes cousaient de nouvelles tentes. L'activité principale de l'été était la construction des canots, avec lesquels les familles remontaient dans le territoire à l'automne, et qui occupait les hommes pendant un bon mois. Quand elle visita Natashquan à l'été 1950, l'anthropologue Eleanor Leacock dessina le plan du campement établi à cet endroit, où l'on peut voir des sites de cuisson extérieure "partagés" par deux tentes et des lieux de travail du bois en retrait du campement ${ }^{33}$.

Dans cet extrait, le ton de voix d'Adèle est plein d'une colère contenue. Un peu plus tôt dans l'entrevue, je lui avais demandé de me faire un croquis du campement d'été à la Pointe. Pendant qu'elle parlait, elle crayon-

29. Pour une explication du mode de vie, voir R. Dominique, «Le cycle annuel des Montagnais de Natashquan", dans Le langage de la chasse..., op. cit., 147-174; J. Mailhot, "La marginalisation des Montagnais», loc. cit., 337; R. Savard, Le rire précolombien..., op. cit.

30. Selon Adèle, les Innus laissaient les perches (montants de bois nécessaires pour monter la tente) pour l'année suivante, mais elles étaient ramassées durant l'hiver par les Blancs qui s'en servaient pour se chauffer.

31. Les makushans sont des fêtes collectives, des festins où la nourriture est partagée.

32. Jusqu'en 1942, les Innus de Nutashkuan se rendaient chaque été à Musquaro, à environ $50 \mathrm{~km}$ à l'est, pour la mission annuelle des Innus de l'Est. Les mariages et baptêmes étaient célébrés à cette occasion. Après l'abandon de Musquaro, les missions avaient lieu dans chaque communauté. Adèle, qui est née vers 1939, a plutôt connu la mission à la Pointe. Sur Musquaro, voir D. Gagnon, Deux cents ans de pèlerinage..., op. cit. Voir aussi les récits recueillis par S. Vincent et J. Bacon, «Musquaro: Récits et souvenirs des Innus..." o, op. cit.

33. Eleanor Leacock, "Campement principal d'été des Nutahkuan Innuat, 1950 », cité dans Richard Dominique, Occupation et utilisation du territoire par les Montagnais de Natashquan (rapport de recherche, Village des Hurons, Conseil Attikamek-Montagnais, 1983), 92. 
nait agressivement ${ }^{34}$, et me racontait des anecdotes sur l'envahissement de ce lieu par les «Blancs ${ }^{35}$ »: les pêcheurs de morue venaient jeter leurs déchets devant la plage, les villageois envoyaient paittre leurs vaches qui effrayaient les Innus ${ }^{36}$, les riches avaient un droit de pêche exclusif sur le saumon (nous y reviendrons). Son rire final, un peu amer, plutôt nerveux, est revenu à plusieurs autres occasions où elle avait parlé de racisme. Pour Adèle, la Pointe semblait associée à la fois à sa petite enfance, à un mode de vie aujourd'hui disparu et à un certain sentiment de dépossession.

Peut-être influencée par les photographies que j'avais vues du campement estival des Innus de Nutashkuan prises dans les années 1950, notamment celles de l'infirmière Pauline Laurin, j'avais moi aussi l'impression que c'est à la Pointe que les Innus s'installaient. Mais quelques jours après cette entrevue avec Adèle, je suis entrée en contact avec une autre version de l'histoire. Selon cette version, c'est près de la Petite rivière Natashquan, au village blanc, que les Innus s'installaient l'été:

On n'était pas beaucoup avant, d'après ce que j'ai appris. Pis là où est-ce que, où est-ce que la réserve se situe en ce moment, on n'était pas installés ici. On était installés à côté... ben pas loin du village de Natashquan, là-bas, mais comme... tsé on remontait... tsé ousqu'y a le pont, là, ben on remontait un peu la rivière, pis on était comme installés comme un petit peu plus loin. Ça a l'air qu'on était installés là, pis on était pas beaucoup. Pis c'est par après, je crois, que... que, je sais pas qui exactement, mais y... y nous ont installés ici. [...] C’est parce que j'ai travaillé avec... j'ai travaillé avec une madame, là, que elle ça fait longtemps qu'elle est ici, c'est une Blanche, pis elle me racontait avant que... c'est elle qui m'a raconté ça, que avant les Innus y étaient pas où est-ce qu'on est ici, mais que... là-bas, là, plus loin, à côté de Natashquan, là. (Charles, 20 ans)

À l'inverse de celui d'Adèle, le récit de Charles, un jeune de vingt ans qui a vécu longtemps à l'extérieur de la réserve, est un récit de seconde main, qui lui a été raconté par une Blanche du village voisin de Pointe-

34. Alistair Thomson parle des symptômes physiques d'un traumatisme qui sont perdus à l'écrit, notamment le rire et les gestes de nervosité. A. Thomson, Anzac memories: living with the legend (Melbourne, Oxford University Press, 1994), 10.

35. J'ai choisi d'utiliser ce terme parce que c'est celui utilisé dans les récits et même dans les archives. Il fait référence tour à tour aux premiers colons, commerçants, agents des Affaires indiennes, enseignantes, missionnaires et villageois qui ont été et sont toujours en contact avec les Innus.

36. Pierre Frenette et Bernard Landry mentionnent qu'il y avait environ 50 animaux dans les années 1940, «dans la plaine qui s’étend jusqu’à Pointe-Parent». P. Frenette et B. Landry, Natashquan... le goût du large (Montréal, Nouvelles éditions de l'arc, 2005), cités dans Annie Bourgeois, Les relations interculturelles entre les Autochtones et les Allochtones du Québec : étude de cas des communautés de Nutashkuan et de Natashquan, mémoire de maîtrise (sociologie), UQAM, 2011, 66. 


\section{Figure 2}

\section{Croquis des lieux mentionnés}

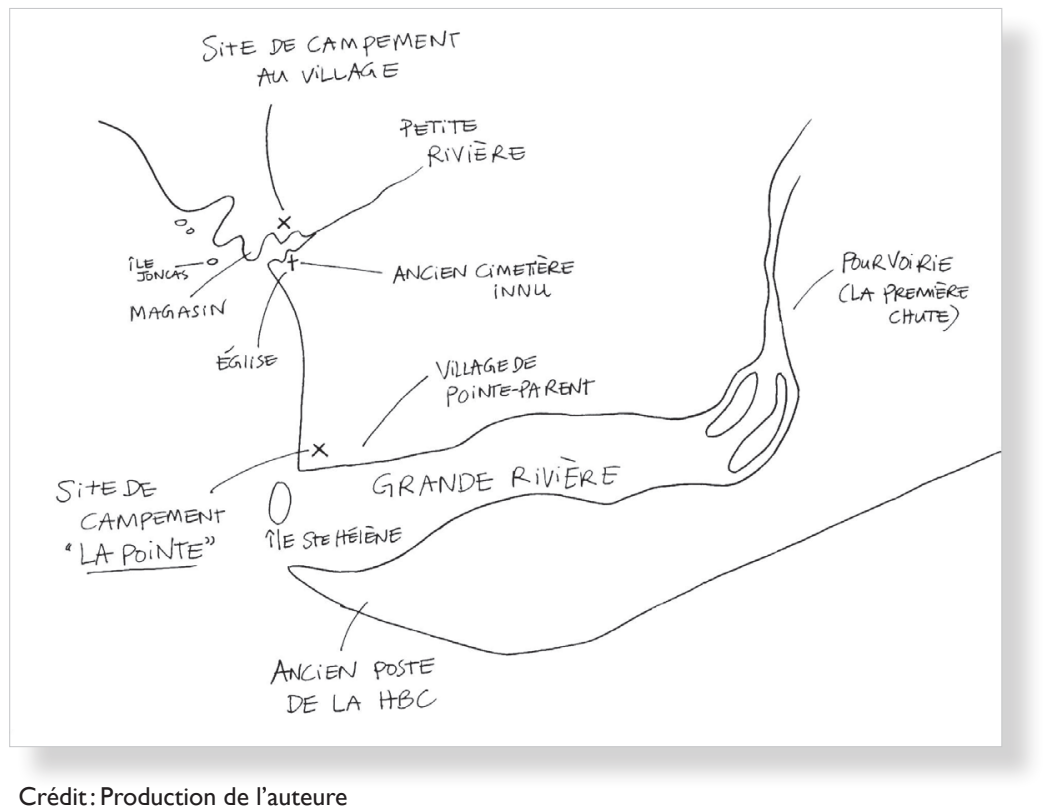

Parent ${ }^{37}$. Il est marqué par une certaine incertitude, par l'usage du conditionnel, par des "ça a d'l'air». J'ai aussi entendu cette version de l'histoire par Olivette McCarthy, une résidante de Pointe-Parent originaire de Rimouski, qui m'a raconté avoir entendu dire que les Innus s'installaient dans un endroit qu'on appelait «le pré», au bord de la Petite rivière Natashquan. Une femme innue m'a aussi dessiné le croquis d'un vieux cimetière situé près de la Petite rivière Natashquan, derrière l'église, où les Innus étaient enterrés. On peut encore y voir quelques tombes. Ce lieu est aujourd'hui le village des Blancs.

En entrevue, Adèle a elle aussi évoqué ce lieu de campement, situé à environ $6 \mathrm{~km}$ de la Pointe. Elle m'a raconté que les Innus suivaient la Petite rivière Natashquan au printemps pour redescendre à la mer; c'était un chemin plus rapide que de longer la Grande rivière Natashquan, et ils arrivaient ainsi directement au village de Natashquan, où ils échangeaient

37. Ce tout petit village est littéralement accolé à la Pointe - aujourd’hui la réserve - au point que l'on confonde avec lui le nom de la réserve, qui est souvent nommée à tort "Pointe-Parent». À force d'agrandissements de la réserve, les maisons de la réserve ont rejoint le village blanc. 
leurs fourrures et s'approvisionnaient au magasin général ${ }^{38}$. Adèle raconte que dans son enfance, les Innus s'installaient à la Petite rivière à la fin de l'hiver; ils y étaient à l'abri dans la forêt et attendaient que les conditions soient plus clémentes pour déménager à la Pointe, qui est un espace dégagé où il y a beaucoup de vent. Mais dans sa description de la Petite rivière, il n'y a jamais autant de vie et d'activités. Pour elle, ce lieu semble avoir été un lieu temporaire, alors que la Pointe était «le» lieu de rassemblement estival.

Par ailleurs, le récit d'Adèle du campement de la Petite rivière était teinté de la perception que les Innus n'y étaient pas les bienvenus. Au printemps, selon elle, ils s'installaient à un endroit où ils étaient tolérés, et pas nécessairement là où ils auraient voulu s'installer:

Comme je disais aux... aux gens d'ici, parce que, des fois y avait toujours... On s'entendait pas avec les gens du village, hein, pis... (pause) Pis, les petits Blancs nous agaçaient tout le temps. Pis... des fois les Blancs y... Y pouvaient pas passer sur le terrain, où est-ce qu'y, tsé où est-ce qu'y est l'hôtel, là... L'hôtel, là où Marie-France travaille, là. (pause) Nous autres on arrivait toujours par là pour descendre au village. Avec nos traîneaux, nos bagages, pis...

Pis eux autres, qu’y aimaient pas ça! «Ah, les maudits Indiens!» (rire) «Les maudits Indiens, y vont toute encore salir, y vont s'installer là-bas!», pis... Fait que, on s'installait à l'autre bord du village. Dans le bois, là, on mettait nos tentes, là, en attendant que ça dégèle ici, à la Pointe. (Adèle Bellefleur)

Dans ses souvenirs, le père oblat Jean Fortin, premier prêtre résident de la communauté, fait écho à cette histoire. Il rapporte les paroles d'un Blanc de Natashquan, selon qui les Innus inspiraient de la crainte quand ils arrivaient au village "avec tout leur attirail». Des histoires circulaient sur le fait qu'ils étaient "malins» et qu'ils volaient. Ils menaient un mode de vie différent et «mystérieux» qui n'était pas compris ${ }^{39}$.

Dans les archives, des lettres de Blancs qui ont visité le village à cette époque confirment aussi la présence d'une volonté de garder une distance entre les deux groupes. À l'été 1946, l'agent local des Affaires indiennes, Patrick Maloney, visita la communauté de Natashquan en compagnie du père oblat Joseph Décarie, basé à Betsiamites. À l'époque, les seuls missionnaires permanents sur la Côte-Nord étaient basés à Betsiamites et à

38. Le poste de traite à la Pointe avait été fermé des années auparavant à cause de l'érosion des berges. BAC, RG10, vol. 3190, dossier 471,601, C. C. Parker, «Report on Mingan, Seven Islands \& St Augustin Agencies », 23 juillet 1915, p. 18-19.

39. Jean Fortin, Coup d'œil sur le monde merveilleux des Montagnais de la Côte-Nord, 1954-1980 (Wendake, Institut culturel et éducatif Montagnais, 1991), 136-137. 


\section{Figure 3 \\ Jean-Baptiste Bellefleur arrivant au village de Natashquan avec son traîneau}

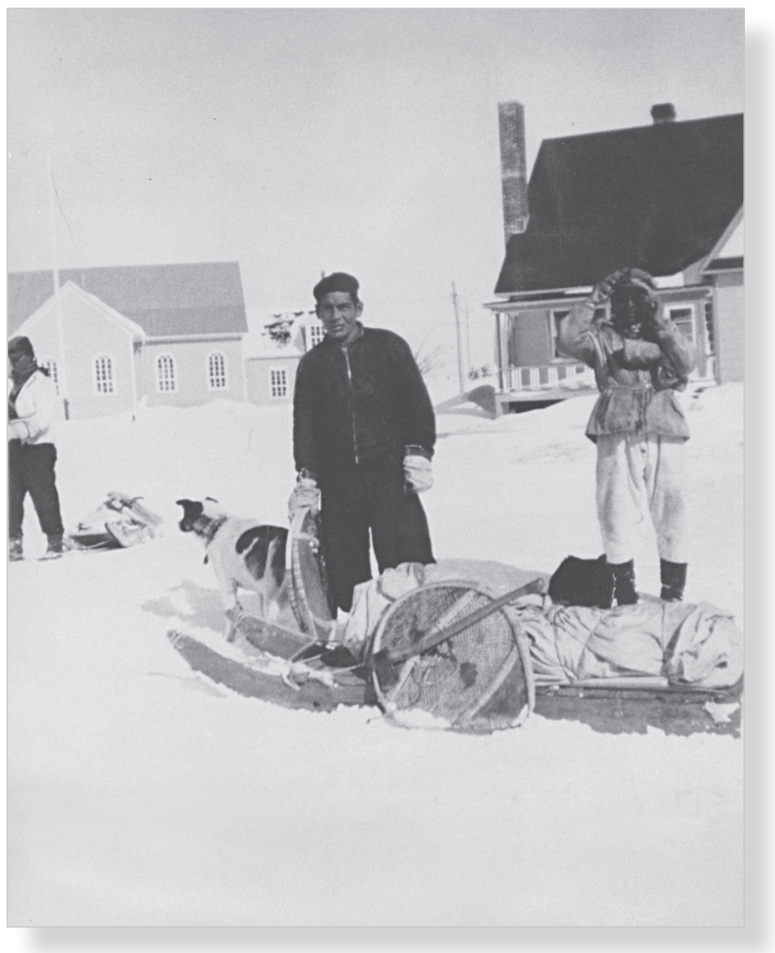

Crédit: Photo par le Père Jean Fortin [?]

Avec l'aimable autorisation de Sylvie Malec, école Uauitshitun,

Nutashkuan

Sept-Îles; quant aux agents des Affaires indiennes, c'étaient des commerçants ou notables de la région, comme Maloney, commerçant de HavreSaint-Pierre, qui assumaient ce rôle à temps partiel. Les deux hommes étaient convaincus qu'il fallait établir une réserve pour Natashquan et s'étaient entendus lors de leur visite estivale sur un terrain qu'ils voulaient demander au département des Affaires indiennes. Pour des raisons pragmatiques, ils préconisaient un site près du village blanc, où les commodités seraient plus faciles à installer ${ }^{40}$; on parlait d'ouvrir une école et un dispensaire et de construire des maisons, des réalisations qui seraient

40. Il n'y avait pas de route entre Natashquan et Pointe-Parent/Nutashkuan avant 1962. Jean Desmeules, «Natashquan, localité sous-développée de la moyenne Côte-Nord», Revue canadienne de géographie, 17, 1-2 (1963): 49. 
facilitées en profitant des infrastructures déjà existantes au village ${ }^{41}$. Mais quelques mois après leur visite, Maloney écrivait à Décarie:

Je dois vous informer que la municipalité de Natashquan s'oppose à ce que les Indiens s'établissent à proximité du village. Dans ce cas, il n'y aura rien de fait sur les terrains dont il était parlé lors de votre passage ici l'été dernier. Quand l'inspecteur du Département viendra le printemps prochain, il faudra qu'un endroit soit choisi afin d'y établir une réserve. Pour cette année je crois que les sauvages devront rester au même endroit, à la grande rivière Natashquan ${ }^{42}$.

Trente ans plus tôt, en juin 1915, le missionnaire eudiste ${ }^{43}$ Tortellier s'était rendu au village de Natashquan pour «faire la mission » aux Innus. Ceux-ci se trouvaient alors à la Petite rivière, mais isolés sur une île :

Je séjournai plusieurs jours encore à Natashquan, pendant lesquels le fait saillant fut pour moi la petite mission que je fis au groupe indien de l'endroit. Les Montagnais vinrent camper sur une île boisée à peu de distance de l'église; on les mit là, afin d'empêcher leurs nombreux chiens de dévaster les propriétés canadiennes ${ }^{44}$.

En fait, une situation semblable d'exclusion physique était probablement en cours dans toutes les communautés innues de la Côte-Nord. Dans le village voisin de La Romaine, où il y a également un village blanc voisin d'une communauté innue, le père oblat Alexis Joveneau rapportait en 1953, dans une longue lettre à son supérieur Lionel Scheffer, ses impressions sur les relations entre les deux communautés:

Les Indiens sont rejetés par les Blancs, pas en paroles, mais en faits. Il y a 4 ans, ils ont été écartés de force du village au milieu duquel ils campaient. Il existe un chemin (de 6 planches de large) qui traverse tout le village. Ce chemin est également utilisé par les Indiens qui se rendent de la réserve à la H.B.C. [Compagnie de la Baie d'Hudson] ou à l'école. Certains voudraient, pour empêcher les Indiens de l'utiliser, lancer le projet d'un chemin qui partirait de la réserve à la H.B.C., en contournant le village par derrière!... Idée qui ne se réalisera pas, mais montre la mentalité ${ }^{45}$ !

41. Archives historiques des Missionnaires Oblats de Marie-Immaculée au Canada (AHMOIM), Archives Deschâtelets, HRI 3.C73R 15, J. Décarie à J. O. Plourde, 21 octobre 1946.

42. AHMOIM, Archives Deschâtelets, HRI 4.C73R 2, P. Maloney à J. Décarie, 22 février 1947.

43. Les Eudistes avaient remplacé les Oblats sur la Côte-Nord entre 1912 et 1946, car les Oblats avaient été jugés trop complices des Innus. Denis Gagnon, «Les Innus de la Basse-Côte-Nord et la mission catholique de Musquaro (1800-1946)", Recherches amérindiennes au Québec, 32, 2 (2002): 51-52.

44. AHMOIM, Archives Deschâtelets, LCB 6001.L12E 11, A. Tortellier, «En Mission Montagnaise», 28 décembre 1915.

45. AHMOIM, Archives Deschâtelets, LCB 6288.L12L 013, A. Joveneau à L. Scheffer, 28 juillet 1953. 
Si les archives et les récits tendent à confirmer une volonté de garder une distance entre les deux groupes, il existe à Natashquan un certain mythe concernant les bonnes relations entre les Innus et les Blancs. Au moment de l'ouverture de la région au peuplement libre au milieu du $\mathrm{XIX}^{\mathrm{e}}$ siècle, des pêcheurs acadiens venus des îles-de-la-Madeleine s'installèrent à la Petite rivière Natashquan où ils fondèrent un village en 1855 . On raconte que chacun vivait de ressources différentes - les Innus du saumon, les Acadiens de la morue - dans une relative entraide ${ }^{46}$. Or, le récit du site de campement traditionnel fait plutôt ressortir des tensions pour l'occupation et le partage des espaces entre Blancs et Innus, des tensions qui s'incarnent dans des lieux précis du village.

L'église, par exemple, est un lieu important dont on m'a souvent parlé. Avant d'avoir une église dans la réserve, les Innus fréquentaient celle de Natashquan, mais il semble qu'ils devaient rester derrière, au fond de l'église, une situation qui s'est répétée quand le père Jean Fortin s'est mis à dire la messe à l'école de la réserve; il m'a raconté s'être fâché en demandant aux Blancs de laisser la place «aux Premiers», qui devaient s'asseoir par terre parce que les Blancs prenaient tous les bancs. La première chose que les Innus lui demandèrent, dès son arrivée en 1958 , c'est la construction d'une église à eux, sur la réserve ${ }^{47}$. Les Innus de Nutashkuan ont construit l'église bénévolement, un fait qui semble avoir été assez exceptionnel, si on en croit la correspondance des Oblats ${ }^{48}$. On m'a parlé avec fierté de la construction de cette église et du travail bénévole des hommes. Joseph Tettaut, 62 ans, m’a raconté que son grand-père travaillait sur son canot le matin et allait travailler à la construction de l'église l'après-midi.

Le magasin est un autre lieu où s'incarnait le racisme. Les Innus y étaient moins bien servis, moins bien traités que les Blancs. Quand elle m'en a parlé, Adèle a ponctué ses phrases de pauses, et du même petit rire amer et nerveux que j'ai déjà évoqué, en donnant au marchand une grosse voix :

46. Voir entre autres A. Bourgeois, Les relations interculturelles entre les Autochtones et les Allochtones..., op. cit., 36-37.

47. J. Fortin, Coup d'œil..., op. cit., 7-8. Le père Alexis Joveneau, dès son arrivée à La Romaine, constatait aussi qu'il fallait deux églises pour les deux groupes, car les Blancs refusaient de se rendre à la messe des Innus. AHMOIM, Archives Deschâtelets, LCB 6288.L12L 013, A. Joveneau à L. Scheffer, 28 juillet 1953.

48. Le coût de la chapelle était estimé à 50000 \$, mais elle n’avait coûté que 14000 (pour les matériaux). Archives des Oblats à Richelieu, 5D1/34-15, Jean Fortin, "Construction église mission Natashquan", 1961-1962. "Pas un sous [sic] de salaire sur les travaux, tous ont fait leur part de leur bras et de leur gousset »: AHMOIM, Archives Deschâtelets, HRI 4.C73C 12, J. Fortin à père Renaud, 29 octobre 1961. 
Une fois j'étais avec mon grand-père, pis on attendait longtemps pour se faire servir. (pause) Pis... quand le bateau arrivait, là, c'était eux autres, tout le temps, y avait tout le temps des commandes pour, telle famille Landry, ça c'est, "touchez pas à ça!», toutes les paquets étaient faits, y’étaient... y’étaient à part, là. Toutes les bonnes choses, pis tout ce qui restait, là, des fois des patates qui étaient pourries, là, pis... y servaient ça aux Indiens. Pis... (pause) Ah oui je m'en souviens, très très bien (rire). [...] Pis nous autres on attendait que nos parents soient servis, là... Pis là quand y étaient servis, enfin, vers $5 \mathrm{~h}$, quand les magasins fermaient, là, c'était vite, vite, là, ils choisissaient même pas les... les patates, si y’étaient pourries, y mettaient ça dans des sacs, là, « envoye, allez-vous en avec ça, là ! Le magasin ferme, là !» Pis nous autres on partait avec ça, on entassait tout ça dans les traînes, les traîneaux, pis on partait avec ça au campement. Des fois quand on arrivait là-bas, y déballaient les, les choses, c'était toute gelé, c'était toute pourri, ah! Ah oui, ça arrivait, très très souvent. Les... les Indiens, les Innus, étaient toujours... servis à part. (pause) Ouais. Ah oui je m'en souviens très bien, y en a beaucoup qui disent ça, ceux qui s'en souviennent. Même ma sœur elle a dit, une fois elle est allée chercher des patates. Pis elle dit que y avait eu un gros sac, pis il l'a chargé, tsé, pas mal, là [c'était cher]... pis elle a dit ok, je vais l'acheter, pis quand elle est arrivée à la maison, y avait juste des bonnes sur le dessus, les autres étaient toutes pourries. (Adèle Bellefleur)

Cette crainte ou réticence des Blancs à voir les Innus s’installer dans leur village s'inscrit dans un phénomène de distanciation physique et sociale, que certains participants - blancs - ont associée à une tendance "naturelle» à se regrouper entre semblables ${ }^{49}$. Mais au-delà des réactions individuelles des villageois, il est important de rappeler la présence d'un racisme institutionnalisé. Comme le souligne Constance Backhouse dans son essai Colour-Coded, le racisme ne surgit pas de façon isolée ; il se situe " in the systems and structures that girded the legal system of Canada's past ${ }^{50}$ ». L’image de l'Indien véhiculée dans les années 1940-1950, d'un individu paresseux, sale, inférieur, qu'il fallait éloigner, s'était construite depuis le $\mathrm{XIX}^{\mathrm{e}}$ siècle ${ }^{51}$, et elle influençait certainement la réaction des personnes en contact avec les Innus. Les récits des Innus de Nutashkuan

49. Une résidente de Pointe-Parent m’a parlé du fait que les enfants des deux groupes ne jouaient pas nécessairement ensemble, mais elle associait cela à une ignorance mutuelle plutôt qu'à du racisme. Selon le père Fortin, ce serait "une différence culturelle» qui aurait incité les Blancs et les Innus à vivre côte à côte sans trop se mêler.

50. Constance Backhouse, Colour-Coded: A Legal History of Racism in Canada, 1900-1950 (Toronto, University of Toronto Press, 1999), 15. Voir l'introduction plus globalement.

51. John Sutton Lutz, Makúk: A New History of Aboriginal-White Relations (Vancouver, UBC Press, 2008), 31-47. Le racisme était basé non seulement sur des phénotypes mais sur des caractéristiques sociales. 
tendent à montrer qu'une ségrégation était à l'œuvre, un phénomène souvent ignoré dans l'histoire canadienne ${ }^{52}$. La Loi sur les Indiens, qui se base sur la création d'un espace physique et légal distinct qui place les Indiens en marge de la société dominante, allait concrétiser cette exclusion territoriale et économique.

\section{« LE CHEF A MESURÉ LA RÉSERVE AVEC SES PAS »: LE CHOIX DU SITE, ENTRE FIERTÉ ET INCOMPRÉHENSION}

Moi: Pis votre grand-père, est-ce qu'il vous a expliqué... est-ce qu'il vous a raconté comment ça s'est passé, son installation sur la réserve? Comment y est passé du mode de vie dans le bois à... au mode de vie sur la réserve?

AI: Mon grand-père me disait toujours... (pause) D’abord les gens étaient pas scolarisés dans le temps. Quand tu demandais, c'est quoi que tu veux comme territoire, là... Le fonctionnaire arrivait, y dit c'est quoi... [...] il dit «combien vous êtes ici?», combien qu'on est ici, les gens disaient, "combien qu'on est ici?», bon, on est peut-être vingt, vingt familles. Bon, y dit, "on prend le territoire jusqu'à... jusqu'à Natashquan ", par exemple. Ben... Voyons la réalité, ici, t'as $1 \mathrm{~km}$ par $3 \mathrm{~km}^{2}$ de réserve. Pis c'est les anciens qui ont pris ça. Parce que dans leur principe, le régime territorial n'a jamais existé pour eux autres. Pour eux autres, c'était juste un campement, dans le temps. Dans le temps. Mais le gouvernement en a profité, de dire, «bon maintenant c'est votre réserve. C’est votre réserve, là, vous allez rester là.» [...]

Moi : Pis ce fonctionnaire-là qui est venu ici, qui a rencontré, ça veut dire qu'il est venu un été quand tout le monde était au campement, pis y a parlé avec les Anciens, selon vous, là, c'est comme ça que ça s'est passé?

AI: Ouais, les Anciens.

Moi: Pis c'est qui, ce fonctionnaire-là, on sait-tu son nom?

AI: Non non, mais je sais que c'était Sylvestre Bellefleur, dans le temps, là... Sylvestre Bellefleur, c'est le premier chef de la communauté. [...]

Moi: Y a-tu quelqu'un qui se souvient de ça, ou ben...?

AI: Ça doit être dans des archives. Dans les affaires, au niveau du ministère des Affaires indiennes, la meilleure façon d'aller chercher, c'est ça. S'ils l'ont pas, ça doit être à Ottawa. (Antoine Ishpatao, 57 ans)

Pour moi qui m’intéressais au processus de création de la réserve, le récit d'Antoine Ishpatao est venu alimenter une image ancrée dans mon 
imaginaire depuis l'enfance. L'idée de cette rencontre entre les Anciens et les fonctionnaires m'a rappelé les vieux westerns hollywoodiens, les bandes dessinées de Lucky Luke et les gravures de mes livres d'histoire représentant la signature des traités entre le gouvernement canadien et les Cris-des-Plaines ${ }^{53}$. J'imaginais un face-à-face solennel entre les Innus et les représentants du gouvernement venus négocier la cession des terres, et qui aurait scellé le sort de la communauté.

D'autres versions de cette rencontre entre les fonctionnaires des Affaires indiennes et le chef de la communauté, accompagné ou non des Anciens, selon les versions, m’ont été racontées. Au cours de plusieurs rencontres informelles, mes interlocuteurs ont précisé que le chef aurait mesuré avec ses pas la dimension de la réserve : il aurait marché environ 100 pas dans chaque direction, et il aurait dit: «ça, ça serait assez». À l'époque, la population de la «bande» de Natashquan était d'environ 140-150 personnes $^{54}$, ce qui correspond grosso modo à la "vingtaine de familles » du récit d'Antoine ou à la liste des résidents élaborée par l'anthropologue Eleanor Leacock à l'été 1950 , où il y a 23 tentes ${ }^{55}$. Comme le dit Antoine, ce territoire "de 100 pas de côté», déterminé par le chef, était probablement suffisant pour y établir un campement d'été, ou correspondait peut-être même au campement déjà existant.

Cette image, bien que précise, restait très incomplète. Où et quand cette rencontre avait-elle eu lieu? Qui y était présent? Y avait-il des interprètes? Quelle sorte d'entente avait été prise? Y avait-il un document signé qui en attestait? Existait-il des photos de l'événement?

Sur les conseils d'Antoine, j'ai cherché dans les archives des traces de cette rencontre pour corroborer les récits. Premier constat: il n'y a pas d'archives officielles dans la communauté. Au Conseil de bande de Nutashkuan, je n'ai pas pu trouver de documents liés à la création de la réserve, ni même de vieilles photographies. En entrevue et dans des rencontres informelles, mes interlocuteurs ont souvent évoqué des destructions de documents publics ou privés: inondation dans le sous-sol du Conseil de bande, incendies accidentels de maisons, destruction de la

53. Dans le livre de Sylvie Vincent et Bernard Arcand, L'image de l'Amérindien dans les manuels scolaires du Québec, ou Comment les Québécois ne sont pas des sauvages (LaSalle, Hurtubise HMH, 1979), le chapitre 9 sur «Les droits territoriaux» fait référence à cette vision idyllique de la création des réserves de l'Ouest grâce à des «négociations» et des «ententes» avec les Autochtones (p. 255-256).

54. En juin 1947, l'agent des Indiens Patrick Maloney évaluait la population à 147 personnes. BAC, RG10, vol. 9034, «Report of Discussions at Conference of Indian Agents from Province of Quebec and the Maritimes, June $9^{\text {th }}, 10^{\text {th }}, 11^{\text {th }}, 12^{\text {th }}, 1947 »$, p. 38 .

55. E. Leacock, "Campement principal d'été des Nutahkuan Innuat, 1950 », loc. cit. 
vieille école, négligence d'un curé qui aurait jeté de vieilles photographies accumulées par son prédécesseur, vandalisme dans les bureaux du Conseil de bande dans les années 1970 à la suite duquel des archives seraient parties au vent: les histoires ne manquaient pas. Ces divers événements auraient mené à la disparition de photos, de baptistères, de listes de classes et de documents liés à la création de la réserve.

Malgré mes recherches, je n'ai pas trouvé dans la réserve de documents datant d'avant les années 1980, alors que la création de la réserve remonte aux années 1950. Il est également probable que les documents en question n’aient jamais été laissés dans la communauté; j’avais plus de chances, comme le supposait Antoine, de trouver ce que je cherchais dans les archives officielles, à des centaines de kilomètres de la réserve. L'accès aux archives reste un problème pour beaucoup de communautés autochtones, qui n'ont souvent ni le temps ni la possibilité d'entreprendre elles-mêmes des recherches dans les centres d'archives nationales situés dans les grands centres $^{56}$.

Dans les archives des Affaires indiennes, à Ottawa, je n'ai pas trouvé non plus de traces de la rencontre évoquée dans les récits. Je cherchais des correspondances ou des discussions entre les agents sur le terrain et leurs supérieurs à Ottawa, mais les archives posaient d'autres problèmes: la quantité gigantesque de documents produits par les Affaires indiennes après la Guerre ${ }^{57}$, les méthodes de classement qui rendent difficile l'identification d'une communauté précise, l'accès restreint à certains dossiers. J'ai épluché des milliers de documents d'archives provenant du célèbre fonds des Affaires indiennes (RG10) ainsi que des archives des Oblats, mais j'ai trouvé un seul indice d'une éventuelle rencontre. Dans la lettre au père Décarie citée plus haut, Patrick Maloney laissait supposer qu'une rencontre était prévue à ce sujet: "Quand l’inspecteur du Département [le fonctionnaire du récit d'Antoine?] viendra le printemps prochain, il faudra qu'un endroit soit choisi afin d'y établir une réserve ${ }^{58}$. " La lettre ne mentionne pas si cette «rencontre» devait inclure des membres de la communauté ou pas.

J'ai raconté cette histoire à Sylvie Vincent, anthropologue réputée en histoire orale des Innus et qui travaille avec la communauté de Nutashkuan

56. A. D. Palmer, «Introduction», dans Maps of Experience ..., op. cit., 24.

57. Pour Hugh Shewell, la multiplication des archives des Affaires indiennes après la Deuxième Guerre mondiale est l'un des obstacles majeurs à toute étude sur le sujet. H. Shewell, «Enough to Keep Them Alive" - Indian Welfare in Canada, 1873-1965 (Toronto, University of Toronto Press, 2004), 207-208.

58. AHMOIM, Archives Deschâtelets, HRI 4.C73R 2, P. Maloney à J. Décarie, 22 février 1947. 
depuis 40 ans. Selon elle, il y a peu de chances que les choses se soient passées de cette façon, car règle générale, les réserves étaient choisies ou établies par les fonctionnaires à Ottawa, parfois sur les recommandations des agents locaux ${ }^{59}$. Selon Anne-Marie Panasuk et Jean-René Proulx, c'est Maloney lui-même qui aurait fait la demande d'une réserve pour les Innus de Nutashkuan ${ }^{60}$. Déjà, en 1908, un agent de passage, J. Ansdell MacRae, recommandait que l'on confie à J. E. Tremblay, médecin blanc de Sept-Îles et agent local des Affaires indiennes, le mandat de déterminer quels seraient les meilleurs endroits pour établir des réserves pour les Innus de la Côte-Nord. Pour Natashquan, MacRae parlait d'un site sur la rive nord de la rivière où se trouvaient déjà quelques maisons; ce site se trouvait à proximité des bancs de morue, pêche à laquelle le département des Affaires indiennes voulait convertir les Innus ${ }^{61}$. Nulle part n'était-il fait mention de consulter les principaux intéressés.

Pourtant, dans les récits, cette consultation est importante. Elle sert à insister tant sur l'autorité du chef et des anciens que sur leur incompréhension de ce qui était en train de se passer. Le récit de François Bellefleur, ancien chef de la communauté, fait écho à celui d'Antoine :

La façon dont... que ça s'est décidé, l'agent des Affaires indiennes, lui... C'est un agent, évidemment, y avait... J'ai su qu'il avait demandé à des aînés qui voulaient statuer une communauté près de l'aéroport, à Natashquan, où est-ce que... Je pense qu'il voulait plus les éloigner plus, où est-ce qu'il pouvait y avoir un secteur économique qui pouvait se passer, comme la pêche au saumon, là, c'est... Nous, là, c'était pas, on le voyait pas de façon économique, c'était pour nous nourrir de cette ressource-là. Pis en plus ici on est dans un endroit où est-ce qu'il y a une pointe, où est-ce que la chasse se fait, pour la chasse... au canard, pis la chasse au loup-marin [phoque], tout ça, là, qui va... On pouvait mettons, facilement prélever dans ce secteur-ci, c'est ici que les aînés ont signifié d'établir une communauté autochtone. Pis ils étaient en

59. Communication personnelle, 12 novembre 2013. Sur le processus d'attribution de certaines réserves en collaboration avec les nations autochtones, voir les séjours de Gilbert Malcolm Sproat en Colombie-Britannique : Cole Harris, «The Joint Indian Reserve Commission, 1876-1878» et «Sproat and the Native Voice, 1878-1888", dans Making Native Space: Colonialism, Resistance, and Reserves in British Columbia (Vancouver, UBC Press, 2002), 104-135 et 136-166.

60. A.-M. Panasuk et J.-R. Proulx, La résistance des Montagnais à l'usurpation des rivières à saumon..., op. cit., 213. Malheureusement, ces documents utilisés par Panasuk et Proulx n'ont pas pu être retracés dans les archives du RG10; une information manquante dans la référence ou la réorganisation des archives depuis leur consultation par Panasuk et Proulx en sont les causes probables.

61. BAC, RG10, vol. 3048, dossier 237,660, pt. 18, J. Ansdell MacRae, «Bersimis and Mingan Agencies - Reports on these Agencies by J. Ansdell MacRae, Inspector of Indian Agencies. Included are reports on Various Bands Living on the North Shore of the St. Lawrence River, the Nascopies (Maskopees), Esquimox and Montagnais Living at Seven Islands, Godbout, Moisie, Musquares, St. Marguerite River, Romaine, Point a Maurier and St. Augustine (Map, Natashquan Census 1908) ", 9 septembre 1908, 22-23. 
désaccord complet pour aller s'installer à Natashquan. Pis je pense que c'est là que... une fois qu'y ont eu, qu'ils ont décidé de rester ici, y ont demandé jusqu'où qu'ils voyaient une communauté. C'est là que je dis que, les gens n'ont pas compris la notion de communauté, une réserve indienne. Un secteur, comme exemple, comme une municipalité, la dimension d'une municipalité. Eux autres, y a des aînés qui ont dit que la dimension de la communauté, c'est toute le territoire qu'on a fréquenté, où est-ce qu'on a parcouru, c'est ça notre territoire autochtone. Mais y a un niveau qui a été créé où est-ce qu'on pouvait établir une réserve indienne, c’est ça. (François Bellefleur, 55 ans)

Un aspect important du récit de la rencontre, sur lequel tous mes interlocuteurs ont insisté, c'est que jamais les aînés n'auraient voulu dire par là qu'ils cédaient le reste du territoire. Pour ceux qui m’ont raconté l'histoire, la conclusion était la même: le chef et les Anciens n'avaient pas compris ce qui était en train de se passer; qu’en désignant un territoire ou en mesurant un carré sur le sol pour signifier où ils souhaitaient s'installer, ils étaient en train de créer une "réserve indienne " où ils devraient désormais vivre à l'année, régis par des mesures contraignantes enchâssées dans la Loi sur les Indiens.

La création des réserves s'inscrit dans un processus de transcrire dans l'espace physique une volonté d'exclure et de contrôler les Autochtones, définie dans la Loi sur les Indiens. Les premières réserves sont apparues sous le Régime français, sur le modèle des réductions d'Amérique du Sud. Le processus s'est accéléré avec la fin des alliances militaires, au début du $\mathrm{XIX}^{\mathrm{e}}$ siècle, puis avec l'expansion de la colonisation vers l'Ouest et la signature des traités numérotés; les réserves faisaient partie d'un processus de «civilisation" et d' "occidentalisation» des Autochtones ${ }^{62}$.

62. Sur la création des premières réserves sous le Régime français, voir entre autres Rémi Savard, "La "réduction" de Sillery 1638-1660: maquette de l'idée de "réserves indiennes" ", Recherches amérindiennes au Québec, 38, 2-3 (2008): 127-131; Larry Villeneuve et Daniel Francis, The Historical Background of Indian Reserves and Settlements in the Province of Quebec (Ottawa, Indian and Northern Affairs Canada, 1984), 5-10. Sur l'orientation de la politique des Affaires indiennes après la fin des alliances militaires au début du XIX e siècle et le paradoxe entre "protéger» et "civiliser», voir J. R. Miller, "From Alliance to "Irrelevance" " et "Reserves, Residential Schools, and the Threat of Assimilation", dans Skyscrapers Hide the Heavens $-A$ History of Indian-White Relations in Canada, $3^{\text {rd }}$ edition (Toronto, University of Toronto Press, 2000), 103-124 et 125-147; John Leslie et Ron Maguire, dir., The Historical development of the Indian Act (Ottawa, Indian and Northern Affairs Canada, 1978), 1-36; Richard H. Bartlett, "False Analogy of Indian Reserves to Traditional Lands", dans Indian Reserves and Aboriginal Lands in Canada: A Homeland - A Study in Law and History (Saskatoon, University of Saskatchewan Native Law Centre, 1990), 65-71. Pour un exemple du discours «bienfaiteur» de l'époque (1861), voir Herman Merivale, «Policy of Colonial Governments towards Native Tribes, as Regards Their Protection and Their Civilization ", dans Olive Patricia Dickason, dir., The Native Imprint, vol. 2 : From 1815: The Contributions of First Peoples to Canada's Character (Canada, Athabaska University Press, 1996), 12-20. 
Sur la Côte-Nord, comme dans d'autres régions éloignées, l’influence des Affaires indiennes a mis plus de temps à se faire sentir. En 1861, on avait créé une première réserve à Betsiamites, où l'on souhaitait rassembler tous les Innus et les convertir à un mode de vie agricole; deux autres petites réserves avaient été créées, dont Sept-Îles en 1906, après vingt ans d'efforts des Innus eux-mêmes pour protéger leur territoire ancestral de l'expansion de la ville ${ }^{63}$. C'est vraiment après la Deuxième Guerre mondiale, avec le développement économique de la région et l'explosion du département des Affaires indiennes, que l'on commença à créer d'autres réserves sur la Côte-Nord. En 15 ans, on est passés de 3 à 7 réserves, toutes de petites dimensions ${ }^{64}$; la volonté de créer de petites réserves visait à offrir un lieu de résidence à partir duquel les Innus pourraient intégrer le mode de vie occidental, et non pas à leur accorder un territoire où ils pourraient pratiquer un développement économique viable ${ }^{65}$.

Paradoxalement (ou pas), la Côte-Nord vivait alors une "ruée vers le Nord», accompagnée d'une forte poussée démographique ${ }^{66}$. Durant ces années de Révolution tranquille, la région s'est construite sur une mythologie nationale basée sur le repoussement des dernières frontières ${ }^{67}$. Or, comme dans le cas de la "vraie» ruée vers l'or, les Innus n'ont pas profité de ce développement économique. Leur présence a été effacée de l'histoire au profit de l'image du valeureux défricheur, bâtisseur de la nation ${ }^{68}$. Il est intéressant de noter que, dans les articles de l'époque, les Innus

63. J. Mailhot, «La marginalisation des Montagnais», loc. cit., 354-357; A.-M. Panasuk et J.-R. Proulx, La résistance des Montagnais à l'usurpation des rivières à saumon..., op. cit., 195-199.

64. Pierre Frenette, "Un univers parallèle: les Autochtones», dans Histoire de la Côte-Nord, loc. cit., 508-513 ; Jacqueline Beaulieu, Localisation des Nations autochtones au Québec: Historique foncier (Québec, Gouvernement du Québec, 1998), 133-155. La réserve de Maliotenam est un peu plus grande parce qu’on projetait d'y regrouper plusieurs communautés.

65. J. Mailhot, «La marginalisation des Montagnais», loc. cit., 354-355; BAC, RG10, vol. 3048, dossier 237,660, pt. 18, J. A. MacRae, "Bersimis and Mingan Agencies... », loc. cit., p. 12-13 ; R. H. Bartlett, Indian Reserves and Aboriginal Lands..., op. cit., 1-4 et 65-71. Ce phénomène des réserves trop petites s'est répété partout au Canada. Voir par exemple Cole Harris, "The Native Land Policies of Governor James Douglas», BC Studies, 174 (2012): 101-122; J. R. Miller, Skyscrapers Hide the Heavens..., op. cit., 221-222; Sarah Carter, Lost Harvests : Prairie Indian Reserve Farmers and Government Policy (Montréal et Kingston, McGill-Queen's University Press, 1990), 50-63 ; J. Leslie et R. Maguire, dir., The Historical development of the Indian Act, op. cit., 18-25 ; C. Harris, «Native Space», dans Making Native Space..., op. cit., 265-292.

66. Jean-Charles Fortin, «La ruée vers le Nord», dans Histoire de la Côte-Nord, op. cit., 425-432.

67. Pour un texte de l'époque, voir Fernand Ouellette, "Manifeste de la Manicouagan », Liberté, 6, 5 (1964) : 342-344, http://id.erudit.oeg/iderudit/59934ac. Sur le lien entre identité québécoise et hydroélectricité, voir C. Desbiens, Power from the North..., op. cit.

68. C'est l'exploitation de l'or par les peuples autochtones de Colombie-Britannique qui a déclenché la ruée vers l'or; de la même manière, la découverte du minerai de fer au Labrador en 1937 est attribuée à Mathieu André, un Innu de Sept-Îles. J. S. Lutz, Makúk..., op. cit., 173-178; «Les mines de fer du NouveauQuébec», La Presse, 15 mai 1950, 4, trouvé dans Archives des Oblats à Richelieu, 5D1/23. Sur un récit de la ruée vers l'or du point de vue des Autochtones, voir Julie Cruikshank, «Confronting Cultural Erasure: 
étaient à peu près absents du portrait de la population nord-côtière ${ }^{69}$. Ils n'étaient que rarement employés sur les grands chantiers ${ }^{70} \mathrm{et}$, dans un journal de Sept-Îles de 1952, on trouve la trace d'un discours sur la nécessité pour les familles canadiennes de développer les terres "inoccupées» du Québec ${ }^{71}$. À Natashquan, au début des années 1950, on se préparait à un boom minier qui, bien qu'il ne soit jamais venu, a probablement incité la création d'une réserve à ce moment précis ${ }^{72}$.

En 1953, on a donc officiellement créé la réserve, sur deux lots cédés précédemment au gouvernement du Canada par le gouvernement du Québec au moyen de lettres patentes. Les lots initiaux, les lots 9 et $11 \mathrm{du}$ Village-du-Poste (aujourd'hui Pointe-Parent), d'une dimension de 8,29 hectares, forment un "carré» de moins de 300 mètres de côté ${ }^{73}$. Bien que légèrement sous-estimée, l’image du chef marchant « 100 pas de chaque côté» offre un ordre de grandeur réaliste des dimensions initiales de la réserve de Nutashkuan.

Bien que je n'aie pas trouvé dans les archives officielles des traces qui corroboraient les récits de la rencontre, ou peut-être même à cause de ce silence des archives, ces récits de la création de la réserve me semblent

Images of Society in Klondike Gold Rush Narratives", dans The Social Life of Stories: Narrative and Knowledge in the Yukon Territory (Lincoln et Londres, University of Nebraska Press, 1998), 71-97.

69. Benoît Brouillette, «La côte nord du Saint-Laurent », Revue canadienne de géographie, 1, 1 (1947): 3-21; Paul Bussières, «La population de la Côte-Nord», Cahiers de géographie du Québec, 7, 14 (1963): 157-192.

70. Jean-Sébastien Boutet, «Développement ferrifère et mondes autochtones au Québec subarctique, 1954-1983 ", Recherches amérindiennes au Québec, 40, 3 (2010): 38-44. Sur l'emploi des Innus dans les grands chantiers, voir aussi BAC, RG10, vol. 8214, dossier 379/1-1-1, pt. 1 et 2; vol. 6926, dossier 379/29-1; vol. 8439, dossier 379/23-4, pt. 3 .

71. "Une stratégie de peuplement s'impose», L'Avenir and Sept-Iles Journal, 30 septembre 1952, 8.

72. R. Dominique, Le langage de la chasse..., op. cit., 188-195. Dans L'Avenir and Sept-Iles Journal, on trouve des titres comme "Natashquan entreprend son $2^{\mathrm{e}}$ siècle sous l'essor minier", 21 juillet 1955, 1, ou "Natashquan verra enfin une route grâce à l'Aconic », 8 septembre 1955, 1. En 1951, l'anthropologue Eleanor Leacock s'informait, au nom des Innus de Natashquan, de leurs droits sur les sables magnétiques qu'ils avaient découverts près de La Pointe en 1951. BAC, RG10, vol. 6982, dossier 379/20-5-6-1, pt. 1, "Mining on the Natashquan reserve». Au cours d'une discussion avec Olivette McCarthy, nous avions aussi conclu que la création de la réserve correspondait assez précisément à l'établissement de la mine.

73. Vente de terrains de 8,29 hectares (lots 9 et 11) le 15 avril 1952 par des lettres patentes en vertu de l'arrêté en conseil provincial n 748 du 12 juillet 1949; création de la réserve en vertu de l'arrêté en conseil fédéral n 476 du 31 mars 1953 . La réserve a été légèrement agrandie par suite de rajouts de terrain en 1954 (6,2 hectares, vente du Québec au Canada le 22 mars 1954, arrêté en conseil fédéral no ${ }^{\circ} 1045$ ) et en 1969 (6,5 hectares, transfert du Québec au Canada, arrêté en conseil provincial 1823 du 17 juin 1969), et d'autres lots de moins de 2 hectares en 1970 et 1993, portant sa superficie totale à 22 hectares, soit un "carré» d'environ $450 \mathrm{~m}$ de côté. Henri Dorion, dir., Rapport de la Commission d'enquête sur l'intégrité du territoire du Québec, 4 tranche: Le domaine indien, volume 4.5: Inventaires des réserves et établissements indiens (Québec, Gouvernement du Québec, 1970), 66; L. Villeneuve et D. Francis, The Historical Background of Indian Reserves..., op. cit., 25-26; J. Beaulieu, Localisation des Nations autochtones au Québec..., op. cit., 150151. Je remercie un évaluateur anonyme pour les précisions apportées au texte original de cette note. 
essentiels à l'histoire. Ils expriment un rapport paradoxal face à la réserve: à la fierté ressentie envers les aînés qui ont insisté pour rester sur le site de leur choix se mêle un sentiment d'impuissance et de tromperie face à des mesures venues "d'en haut» qui ont dépossédé les Innus de leur territoire et de ses ressources. Le lien entre la création de la réserve et un développement économique dont les Innus étaient exclus a été largement abordé dans les récits; c'est ce que nous verrons dans la dernière section.

\section{«NOS PÈRES, ILS POUVAIENT PAS PÊCHER LE SAUMON »: L'EXCLUSION DE L'ESPACE ÉCONOMIQUE}

Pis eux autres [les Blancs], ils pêchaient le saumon comme y voulaient. Pis y disaient, "ça c'est le... commercial, pêchez pas le saumon!» Fait que... Y ont bâti un club, y a un gars, en tout cas, un riche qui est arrivé, y a... Y a acheté la rivière, la première chute, là. «Ici on prend, nous autres, toute la chute, le territoire, on paye ça, pis c'est à nous autres, la pourvoirie.» Fait que y se sont approprié la première chute. Pis là y ont bâti les, les maisons qui sont là. Pis on pouvait même pas aller là, les gens... Comme nos pères, y pouvaient pas aller pêcher le saumon. Fait que, c'est ça y a... Y avaient faite ça en... Avant, là, y pouvaient aller chercher le saumon pis le faire sécher tranquilles. Pis... ça a toute coupé (rire) la pêche. Fait que... eux autres, il fallait attendre qu'y aient fini la saison de pêche pour aller pêcher à leur tour. [... ] Des fois les gens y allaient la nuit, pêcher. Y mettaient un petit peu, de filets, pis y amenaient, pis y passaient au travers des bois avec des grosses charges de saumon (rire). Pour juste, ne pas se faire prendre. C'était comme ça qu'ils fonctionnaient. C'était vraiment... de l'injustice pure. Je m'en souviens quand mon grand-père allait au saumon, là. Pis là... y dormait le jour, pis ma grand-mère disait, [en murmurant] «faites pas de bruit, ton grand-père dort!» Parce qu’y est allé au saumon. Fait que y nous jetait dehors, pour qu'on réveille pas... (rire) Tous les gens du village étaient comme ça. (Adèle Bellefleur)

Bien avant la création de la réserve dans les années 1950, des lois étaient venues limiter grandement l'accès des Innus aux ressources naturelles. J'ai mentionné, dans la première section de cet article, l'importance du saumon dans la vie traditionnelle des Innus. Or, en 1857 et 1858, des lois émanant du ministère des Pêcheries du gouvernement de l'Union ont interdit aux Autochtones de pêcher le saumon sur les rivières, qui furent louées à des particuliers. En 1882, les rivières à saumon passèrent sous contrôle provincial, et le gouvernement réitéra la législation mise en place. Dès lors, il devint illégal pour les Innus de pêcher le saumon sur toutes les rivières de la Côte-Nord, sauf sur la rivière Bersimis ${ }^{74}$.

74. "Acte des pêcheries de 1858 ", 22 Victoria, chap. 86, cité dans R. Dominique, Le langage de la chasse, op. cit., 179. J. Mailhot explique que cette interdiction est survenue après une forme de "surexploitation" 
Devant la famine causée par cette interdiction, les Affaires indiennes réagirent par la distribution de «bons» échangeables dans les postes de la Compagnie de la Baie d'Hudson; ces bons, qui étaient en réalité des rations d'urgence, ont été octroyés aux Innus à partir de la fin du XIX ${ }^{e}$ siècle à la suite de nombreuses lettres de protestation envoyées à Ottawa par les Innus eux-mêmes et par les Oblats ${ }^{75}$. Dans son rapport de 1881, l'agent des Indiens L. F. Boucher rapportait avoir distribué aux Innus les plus pauvres de la farine et du saindoux, ainsi que du sel, pour les encourager à pêcher la morue ${ }^{76}$. En 1880, alors qu'il avait relayé la demande des Innus d'avoir un endroit où pêcher sur la Grande rivière Natashquan, Boucher s'était fait répondre qu'il serait impossible d'accéder à leur demande, parce que la rivière était déjà louée à un certain $\mathrm{O}$. Carbonneau de Berthier; il était moins coûteux pour les Affaires indiennes d'acheter du poisson à ce monsieur Carbonneau que de lui louer un espace où les Innus pourraient pêcher eux-mêmes ${ }^{77}$.

Il semble que les Innus aient pu continuer de pêcher le saumon malgré la législation en place. Selon Sylvie Vincent, il y aurait eu une certaine tolérance des gardes-pêche sur la Grande rivière Natashquan, ce que confirme également le récit du père Fortin, qui avait convenu d'un signal avec un garde-pêche sympathique à la cause des Innus pour les avertir de sa venue ${ }^{78}$. À la mort de ce garde-pêche tolérant, le conflit s'est envenimé et a mené à la "guerre du saumon ", qui s'est soldée par la réappropriation par les Innus de la pourvoirie, le «club» dont parle Adèle, en $1982^{79}$.

du saumon par les pêcheurs canadiens et américains; voir «La marginalisation des Montagnais», loc. cit., 338-342. Jacques Frenette fait aussi allusion à une pêche abusive de la HBC, qui contrôlait les rivières jusqu'en 1852, dans Mingan au XIX ${ }^{e}$ siècle..., op. cit., 24 et 49. Pour une étude complète sur le sujet, voir A.-M. Panasuk et J.-R. Proulx, La résistance des Montagnais à l'usurpation des rivières à saumon..., op. cit. Une situation similaire existait sur la Côte Ouest; voir entre autres J. S. Lutz, Makúk..., op. cit., 149-151; Dorothee Schreiber, " "A Liberal and Paternal Spirit": Indian Agents and Native Fisheries in Canada», Ethnohistory, 55, 1 (2008): 87-118.

75. J. Mailhot, «La marginalisation des Montagnais», loc. cit., 333-334 et 340-341; H. Shewell, «Enough to Keep Them Alive...", op. cit., 42-53, 352 note 23. Shewell rapporte que cette aide d'urgence était insuffisante: en 1872, année de la première distribution de rations, il y eut 47 morts à Mingan à cause de la famine.

76. L. F. Boucher, "Annual Report of the Department of Indian Affairs for the year ended $31^{\text {st }}$ Decembre 1881, part $1 »$ (Dominion of Canada, 1882), 19-20.

77. BAC, RG10, vol. 2126, dossier 24,222, L. F. Boucher, "Regarding the Nataskowan River», 20 septembre 1880; BAC, RG10, vol. 2097, dossier 16,761, échanges entre L. Vankoughnet, W.T. Whitcher et L. F. Boucher, 5 novembre 1879 au 30 janvier 1880 .

78. Sylvie Vincent, «Tradition orale et action politique montagnaises : le cas de la rivière Natashquan », dans William Cowan, dir., Papers of the Ninth Algonquian Conference (Ottawa, Carleton University, 1978), 138 ; J. Fortin, Coup d'œil..., op. cit., 34-36.

79. Sur la "guerre du saumon », voir Gérard McKenzie et Thierry Vincent, «La "guerre du saumon" des années 1970-1980: entrevue avec Pierre Lepage », Recherches amérindiennes au Québec, 40, 1-2 (2010): 
En plus des lois sur la pêche au saumon, d'autres lois sur la chasse au gibier sont venues limiter les droits des Innus sur les ressources alimentaires de leur territoire ${ }^{80}$, notamment la création des réserves à castor, un programme de sauvegarde de l'espèce mis en place dans les années 1950 d'après un modèle développé chez les Cris de la Baie James dans les années 1930. À l'encontre de la volonté des Innus, qui avaient exprimé le désir de protéger l'espèce par un plan global de gestion du territoire ${ }^{81}$, le gouvernement procéda à un morcellement du territoire en terrains individuels enregistrés, où les familles devaient recenser le castor sans le chasser en échange d'un montant forfaitaire de $50 \$$, ou $25 \$$, selon les versions. À partir de 1961, certains territoires de la Côte-Nord furent attribués aux non-Autochtones, faisant de la réserve à castor du Saguenay la seule qui ne soit pas réservée aux Autochtones ${ }^{82}$.

De plus, les animaux se sont graduellement faits plus rares à proximité de la Pointe. Élie Bellefleur, 78 ans, et Jules Wapistan, 53 ans, m’ont raconté qu'à une époque, on pouvait trouver du caribou très proche du site de la réserve, même aussi proche qu'à la montagne Bleue, située à moins de $20 \mathrm{~km}$. Le voyage aller-retour pouvait s'effectuer en deux ou trois jours. Les caribous auraient quitté la zone périphérique de Natashquan à partir de 1957, durant la période de développement économique évoquée précédemment ${ }^{83}$. Dans une entrevue réalisée avec l'anthropologue Richard Dominique vers 1976, Paul Wapistan attribuait la diminution du gibier à proximité de Natashquan à la construction d'une nouvelle route pour l'exploitation forestière, ainsi qu'à l'intérêt grandissant des Blancs pour la chasse et la trappe ${ }^{84}$. Le déclin des stocks de morue

103-111; A.-M. Panasuk et J.-R. Proulx, La résistance des Montagnais à l'usurpation des rivières à saumon..., op. cit.

80. Sur les lois émises au XIX ${ }^{\mathrm{e}}$ siècle, voir Denis Gagnon, Deux cents ans de pèlerinage..., op. cit., 115.

81. BAC, RG10, vol. 9034, Patrick Maloney, «Report of Discussions at Conference of Indian Agents from Province of Quebec and the Maritimes, June $9^{\text {th }}, 10^{\text {th }}, 11^{\text {th }}, 12^{\text {th }}, 1947$ », p. 69: "So no one can use that land, they would like to have a closed season for beaver for about 5 years. They would protect the beaver themselves. They want the country protected for the Band as a whole and not for individuals".

82. Sur la création des réserves à castor, voir P. Charest, «Les stratégies de chasse des Mamit Inuat», loc. cit., 107-128; Toby Morantz, The White Man's Gonna Getcha - The Colonial Challenge to the Crees in Quebec (Montréal et Kingston, McGill-Queen’s University Press, 2002), 145 et suivantes; Sylvie Poirier, "Territories, Identity, and Modernity among the Akikamekw (Haut-St-Maurice, Quebec)", dans Colin Scott, dir., Aboriginal Autonomy and Development in Northern Quebec-Labrador (Vancouver, UBC Press, 2001), 105-106; R. Dominique, Occupation et utilisation du territoire par les Montagnais de Natashquan. ..., op. cit., 130.

83. R. Dominique, Occupation et utilisation du territoire par les Montagnais de Natashquan..., op. cit., $109-113$.

84. Institut Tshakapesh, Fonds Richard Dominique, boîte 2, dossier 4, Entrevue avec Paul Wapistan, s.d. 
a mis une pression supplémentaire sur les autres ressources ${ }^{85}$, et accentué les tensions entre les deux communautés.

J'ai déjà mentionné l'existence, à Natashquan, d'un certain mythe sur les bonnes relations entre les deux groupes. Or, de nombreux récits parlent plutôt de conflits, et d'un partage difficile des ressources. Sur l'arrivée des Madelinots, Antoine m'a raconté l'histoire suivante:

AI: Quand les Madelinots sont débarqués ici, bon, ils [les Autochtones] les ont respectés. Mais y avait toujours des petits conflits internes. C'est la répartition du territoire. Si y avait 100 qui débarquaient, pis t'as fait de la gestion... au niveau des Anciens, la gestion du territoire, y en a 100 qui débarquent, pis $\mathrm{y}$ s'en viennent dans ton territoire, oups, $\mathrm{y}$ a un problème quelque part. C'est un peu le même principe que le gouvernement a. Ils sont tellement nombreux, mais ils veulent aller dans le territoire, mais il y a des Autochtones. Tu comprends... Y a toujours un conflit quelque part, qui... Fait que les négociations sont toujours comme ça.

Moi: Le conflit, c'est le fait que tout le monde veut s'approprier le même territoire?...

AI: Le même territoire, c'est un peu dans ce sens-là. C'est pour ça, faudrait... les accommodements raisonnables, je reviens toujours, on pourrait (l'inverser?) d'une certaine façon, c'est applicable aussi. Peut-être que je vois grand, là, mais quand même. Mais c'est la seule façon, y n'a pas d'autre façon. À moins d'écraser l'autre... Regardons le monde, c'est pas sorcier, tout le monde s'entre-tue pour des droits, pour le pouvoir, l'argent, c'est tellement gros, là !... Je veux pas être un prophète de malheur, là, mais quand même, ça a amené à des conflits mondiaux... Mais quand même, on a notre terre à défendre. On a nos valeurs à défendre. (Antoine Ishpatao)

Dans le choix même du site de la réserve, les récits ont souvent fait mention de la volonté des Blancs de s'approprier les ressources naturelles. Après avoir parlé de la volonté des fonctionnaires d'amener les Innus près de l'aéroport et de les éloigner de la rivière pour garder le contrôle sur les ressources, comme nous l'avons vu dans la section précédente, François Bellefleur a fait un lien entre les réserves et la volonté de contrôle, plus largement:

85. Benoît Brouillette, dans "La côte nord du Saint-Laurent», Revue canadienne de géographie, 1, 2-3 (1947) : 18-19, mentionne un creux de la pêche à la morue entre 1920 et 1940; voir aussi A. Bourgeois, Les relations interculturelles entre les Autochtones et les Allochtones..., op. cit., 64-68. Une carte établie par Tanner en 1944, reproduite dans R. Dominique, Occupation et utilisation du territoire par les Montagnais de Natashquan..., op. cit., 112, montre les territoires de trappe des Blancs tout le long de la Grande rivière Natashquan. 
Moi: Quand ils ont créé les réserves, vous pensez que c'était quoi leur objectif, à l'époque?

FB: Contrôler! C’est... y ont toujours contrôlé, quand y ont déposé la Loi sur les Indiens, ça c'est sûr que... (pause) Quand qu'y ont décidé, c'est, c'est sûr que ça vient de la Reine, la Reine... Il fallait qu'ils aient les droits, ils ont les droits, mais y ont tellement détaillé, pour bien... pour bien... Administrer... (pause) le système, pour qu'y aient toujours le dessus, le contrôle de ça. Parce que c'est quoi le contrôle de ça, c'est l'argent! Encore l'argent. Parce qu'ils sont pas gênés d'injecter des fonds pour étirer pendant des années, c'est sûr, eux autres... au fur et à mesure que les entreprises vont exploiter les ressources, eux autres, y ont des revenus constamment, tandis que l'Autochtone, lui, le seul revenu, là... Il y a pas grand revenu, c'est si, les seuls qu'y peuvent avoir comme revenu, c'est les petites entreprises, une pourvoirie ou quelque chose, c'est... Essaie de te battre, un revenu, une pourvoirie, avec toutes les pourvoiries minières, forestières, à travers le Canada. À travers le Canada. (François Bellefleur)

Adèle a aussi insisté sur le rôle de l'économie et le pouvoir des marchands locaux dans les prises de décision, en ramenant cette idée de «rencontres» qui déterminaient le développement de la communauté:

Les décisions, ça se faisait avec le prêtre, les Anciens, les marchands. C’était les marchands qui tenaient la place comme gouverneurs, c'était très important, leur opinion. C'était eux les monsieurs qui étaient comme le maire. Les curés pis les marchands. Les Affaires indiennes, il n'y en avait pas tellement de formé encore, vu que les Innus étaient tout le temps nomades, ils étaient tout le temps partout. La plupart du temps, les plus vieux [Innus] étaient informés par les sédentaires [les Blancs] des décisions qui étaient prises, ça se faisait de même. Les décisions se prenaient entre les Anciens avec les Blancs qui étaient influents. Quand le monde ont choisi le site, ils ont choisi la Pointe. Quand les Blancs y ont vu qu'il y avait beaucoup de richesses sur ce site-là, le saumon, les oiseaux migrateurs, y ont voulu encore s'approprier de notre Pointe, pis nous autres on savait pas où se ramasser. Les Anciens ont décidé de rester à la Pointe. (Adèle Bellefleur)

Dans ce contexte de partage du territoire et des ressources, il importe de tenir compte du pouvoir des parties en jeu, selon une conception des lieux comme espaces de relations plutôt que comme simples espaces physiques ${ }^{86}$. À Natashquan, le poids démographique des Innus a longtemps joué en leur défaveur: les Innus qui s’installaient à Natashquan avant la création de la réserve étaient beaucoup moins nombreux qu'aujourd'hui, et leur poids 
démographique par rapport aux Blancs était inférieur. Dans les années 1950-1960, il y avait environ 1 Innu pour 3 Blancs $^{87}$.

Aujourd'hui, cette proportion s'est inversée. Les Innus sont près de 1000 à habiter la réserve, tandis que les deux villages blancs combinés comptent moins de 300 habitants. Ce sont désormais les Innus qui font "vivre» les deux villages blancs ${ }^{88}$. Dans leur façon de raconter l'histoire, les Innus ont aussi un pouvoir nouveau. Avec ce changement démographique, il n'est plus de bon ton d'exprimer ouvertement du racisme envers les Innus. Il semble même y avoir une certaine honte, aujourd'hui, à reconnaître que ce racisme ait déjà existé :

$A B$ : Pis quand, j'étais... en troisième année, là, $j$ 'ai commencé à parler le français, là. Pis là j'ai... J’ai pu me défendre un peu, avec les Blancs du village. Pis, quand je leur dis aujourd'hui, là... j'ai dit, les premiers mots que j'ai, les premières phrases que j'ai entendues, que j'ai appris de vous autres, là, c'était... «Mange de la marde!» (rire) Y nous, y nous disaient ça quand on passait, surtout les jeunes. «Hey les maudites sauvagesses, là, passez pas sur le terrain, c'est à nous autres, ça. Allez-vous-en chez vous!» Pis là je dis à, y a une amie, là, que je connais, là, je dis... Vous autres, vous disiez ça hein, quand on passait, je dis, c'était les premières phrases, les premières phrases que j'ai appris en français. «Ah, » elle dit, «c'est pas vrai, parle pas de ça, là !» (rire) Ils veulent pas qu’on leur rappelle...

Moi: Aujourd'hui, quand vous parlez de ça avec elle, ok.

AB : Oui. Oui.

Moi: Elle reconnaît pas que ça s'est passé.

$A B$ : Non. Elle dit, «c'est pas moi certain, moi, ma mère me défendait, ne pas insulter les Indiens, moi j’aimais bien les Indiens !» J'ai dit, comment ça se fait que vous nous jetiez des... des roches, pis toutes sortes d'affaires quand on passait sur votre terrain?, elle dit, on voyait ta mère à la fenêtre, elle disait rien, elle riait. "Oh non non, pas nous autres», j'ai dit oui oui. Ils veulent pas reconnaître ça, hein. (Adèle Bellefleur)

Ces divergences de la mémoire font ressortir ce que la géographe Doreen Massey décrit comme un combat pour l'identité d'un lieu. En se basant sur des interprétations différentes du passé, deux ou plusieurs

87. Benoît Brouillette parle de 140 Innus et de 400 Blancs en 1946, Jean Desmeules de 225 Innus et 675 Blancs en 1963. B. Brouillette, "La côte nord du Saint-Laurent», loc. cit., 8; J. Desmeules, "Natashquan, localité sous-développée..." loc. cit., 46.

88. Sur l'impact économique du rapport démographique, voir A. Bourgeois, Les relations interculturelles entre les Autochtones et les Allochtones..., op. cit, particulièrement 31-39, 76, 98 et suivantes. 
groupes revendiquent leur "droit » à un même lieu, et tendent à raconter un passé qui conforte leur vision du présent et de l'avenir. «The identity of places is very much bound up with the histories which are told of them, how those histories are told, and which history turns out to be dominant ${ }^{89}$." Au-delà du pouvoir d'un groupe ou d'un autre, l'importance de certaines histoires peut changer en fonction du contexte politique. C'est ce que soulignait l'anthropologue Sylvie Vincent, à qui on avait raconté dans le contexte de la guerre du saumon des années 1980 des récits traitant de la rivière, un sujet qui n’avait pas été abordé lors de ses séjours précédents ${ }^{90}$. Ainsi, dans un contexte de revendications territoriales qui s'étire depuis trente ans ${ }^{91}$, il n'est peut-être pas étonnant que les thèmes de la dépossession et de l'exclusion aient pris autant de place dans les récits qui m’ont été racontés.

\section{CONCLUSION}

Parce qu'ils nécessitent un investissement de temps pour créer des relations significatives qui permettent d'aller au-delà du récit dominant, les projets d'histoire orale tendent à être très localisés ${ }^{92}$. Si j'ai été au départ déconcertée par le caractère extrêmement local des récits qui m’étaient racontés, qu'il s'agisse du lieu bien précis du campement ou du chef mesurant la réserve en marchant sur le sol, la valeur de cette spécificité m'est apparue en repensant à Julie Cruikshank. Dans The Social Life of Stories, elle écrivait que "narratives providing the most helpful guidance are inevitably locally grounded, highly particular, and culturally specific ${ }^{93}{ }^{\prime}$. $\mathrm{Au}$-delà de leur caractère personnel, les histoires rapportées ici m'ont permis de comprendre comment des lois et des politiques abstraites et

89. Doreen Massey, "Places and Their Pasts», History Workshop Journal, 39, 1 (1995): 186. Voir aussi D. Massey, "Questions of Locality», loc. cit., 145.

90. Communication personnelle, 12 novembre 2013. Carole Lévesque rapporte le même commentaire dans «La culture entre mémoire et sens... », loc. cit., 106.

91. Au moment d'enregistrer ces récits, la communauté de Nutashkuan, avec celles d'Essipit et de Mashteuiatsh, devaient approuver une entente de principe signée en 2004 avec le gouvernement québécois. En entrevue, l'un des négociateurs de la communauté, Francis Malec, me disait que cette entente devait être entérinée par les communautés par référendum au cours de l'année 2015. Dans leur article sur les traités modernes, Martin Papillon et Audrey Lord affirment plutôt que les négociations sont «en suspens». Martin Papillon et Audrey Lord, «Les traités modernes: vers une nouvelle relation?», dans A. Beaulieu, S. Gervais et M. Papillon, dir., Les Autochtones et le Québec..., op. cit., 343-362.

92. Denis Gagnon parle du «discours officiel» servi aux chercheurs de passage. D. Gagnon, Deux cents ans de pèlerinage..., op. cit., 248; Bruce Granville Miller, dans Oral History on Trial: Recognizing Aboriginal Narratives in the Courts (Vancouver, UBC Press, 2011), 212 p., souligne que l'expertise des chercheurs utilisant l'histoire orale est généralement plus ciblée, souvent limitée à une nation ou à un groupe autochtone.

93. J. Cruikshank, The Social Life of Stories..., op. cit., xii. 
complexes s'étaient appliquées sur le terrain, illustrant que la mémoire individuelle peut avoir une portée beaucoup plus vaste et contribuer à une histoire collective. Ce sont les récits et leurs détails qui ont permis que de grandes zones d'ombre s'éclairent quant à la façon dont on en était arrivé à voir à la Pointe une réserve minuscule où étaient confinés des Indiens qui, quelques décennies auparavant, étaient des Innus se déplaçant à la grandeur du Nitassinan ${ }^{94}$.

Les récits rapportés ici évoquent un sentiment d'exclusion non seulement dans l'espace très local de la communauté mais aussi dans l'espace plus vaste de l'économie et du territoire de la Côte-Nord et du Québec. Ces récits contrastent avec une histoire basée sur les bonnes relations entre Blancs et Innus. Par ailleurs, à ces récits d'exclusion se mêlent des éléments de fierté face aux décisions et à la résistance des aînés, et un attachement à la rivière et au site de la Pointe. Comme dans beaucoup d'autres cas semblables, l'espace d'exclusion qu'est la réserve semble être devenu un lieu d'ancrage et d'appartenance pour les Innus de Nutashkuan ${ }^{95}$.

Il est difficile pour les chercheurs de reconnaître la voix et le rôle des Autochtones et des Innus dans leur propre histoire sans nier la force des structures qui leur ont été imposées. Dans un article qui avait fait beaucoup de bruit, Robin Jarvis Brownlie et Mary-Ellen Kelm avaient souligné le risque de légitimer l'entreprise coloniale en voulant présenter l'«agentivité $^{96}$ » des Autochtones. Plus récemment, Daniel Salée voyait dans une certaine tendance historiographique récente le risque d'un "glissement interprétatif " à trop vouloir ramener les Autochtones au cœur d'un récit historique basé sur la "rencontre ${ }^{97} »$. C’est certainement un défi que de vouloir replacer les Autochtones au cœur de l'histoire sans que cela ne se

94. Nitassinan signifie «notre territoire» en innu. Dans une communication récente, Şükran Tipi mentionnait cependant que Nitassinan n'était que l'un des mots innus utilisés pour parler du territoire, et faisait référence au territoire dans le contexte des négociations avec les deux paliers gouvernementaux. Şükran Tipi, Université Laval, «Entre Nuhtshimits et Nitassinan: narrer et nommer le territoire chez les Pekuakamiulnuatsh Innus du Lac-Saint-Jean », Colloque du Regroupement des géographes du Québec, $83^{\mathrm{e}}$ Congrès de l'ACFAS, 27 mai 2015.

95. R. J. Brownlie et R. Crowe, “"So Yo Want to Hear Our Ghetto Stories?” ... », loc. cit., 210-211. Voir aussi l'exemple du Chinatown de Vancouver: Kay J. Anderson, Vancouver's Chinatown: Racial Discourse in Canada, 1875-1980 (Montréal-Kingston, McGill-Queen’s University Press, 1991), 323 p. Sur le rôle des réserves comme point d'ancrage culturel, voir John Borrows, Seven Generations, Seven Teachings : Ending the Indian Act (West Vancouver, National Centre for First Nations Governance, 2008).

96. L'“agentivité» est une traduction imparfaite du mot anglais "agency», qui fait référence à la capacité d'action et d'adaptation des individus.

97. Robin Jarvis Brownlie et Mary-Ellen Kelm, «Desperately Seeking Absolution: Native Agency as Colonialist Alibi?», The Canadian Historical Review, 75, 4 (1994): 543-556; Daniel Salée, "Les peuples autochtones et la naissance du Québec: Pour une réécriture de l'histoire?", Recherches sociographiques, 51, 1-2 (2010): 151-159. 
traduise par une «absolution» du passé et une négation du rôle mitigé du Québec dans l'histoire des Autochtones icip ${ }^{9}$. En donnant réellement une voix aux acteurs des événements, l'histoire orale peut peut-être nous permettre de nuancer et de complexifier cette histoire récente. Malgré le caractère forcément fragmentaire de tout projet d'histoire orale et les défis liés à cette démarche en contexte autochtone, il me semble important de mettre en œuvre une façon de faire entendre le plus possible ces voix trop longtemps passées sous silence. 